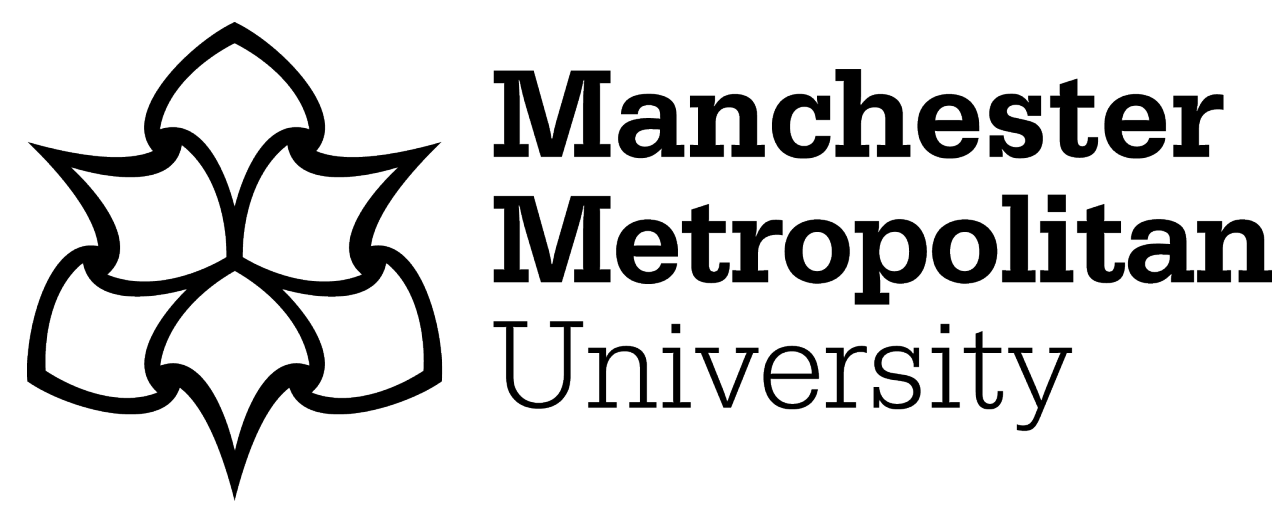

Chen, Hao, Qian, Ling ORCID logoORCID: https://orcid.org/0000-00029716-2342, Ma, Zhihua ORCID logoORCID: https://orcid.org/0000-00022426-3038, Bai, Wei ORCID logoORCID: https://orcid.org/0000-0002-3537207X, Li, Ye, Causon, Derek and Mingham, Clive (2019) Application of an overset mesh based numerical wave tank for modelling realistic free-surface hydrodynamic problems. Ocean Engineering, 176. pp. 97-117. ISSN 00298018

Downloaded from: https://e-space.mmu.ac.uk/622440/

Version: Accepted Version

Publisher: Elsevier

DOI: https://doi.org/10.1016/j.oceaneng.2019.02.001

Usage rights: Creative Commons: Attribution-Noncommercial-No Derivative Works 4.0

Please cite the published version 


\title{
Application of an overset mesh based numerical wave tank for modelling realistic free-surface hydrodynamic problems
}

\author{
Hao Chen ${ }^{\mathrm{a}, *}$, Ling Qian ${ }^{\mathrm{a}}$, Zhihua $\mathrm{Ma}^{\mathrm{a}}$, Wei Bai ${ }^{\mathrm{a}}, \mathrm{Ye} \mathrm{Li}^{\mathrm{b}}$, Derek Causon ${ }^{\mathrm{a}}$, Clive Mingham ${ }^{\mathrm{a}}$ \\ ${ }^{a}$ Centre for Mathematical Modelling and Flow Analysis, School of Computing, Mathematics and Digital Technology, \\ Manchester Metropolitan University, Manchester, United Kingdom \\ ${ }^{b}$ State Key Laboratory of Ocean Engineering, School of Naval Architecture and Civil Engineering, Shanghai Jiaotong \\ University, Shanghai, China
}

\begin{abstract}
This paper presents a numerical wave tank based on the overset mesh approach. Overset mesh is favourable to the moving mesh method owing to its ability to represent complex geometries whilst maintaining a good quality mesh, especially for large amplitude body motions. The numerical wave tank is developed by integrating a generic overset mesh functionality with an efficient wave generation library in OpenFOAM ${ }^{\circledR}$. A series of benchmark test cases, including 2D regular waves interacting with a floating cylinder and boxshaped body with superstructures, $2 \mathrm{D}$ water entry of a rigid wedge and a ship hull section as well as the heave decay of a point absorber wave energy converter and the lifeboat motion in regular waves, are carried out to evaluate the capabilities of the developed numerical wave tank. The computed solutions agree well with the experimental data and other reference results reported in the literature, which demonstrates the capability of the numerical wave tank for modelling flow around structures with complex geometries under various wave conditions. The parallel efficiency of the solver, effects of the overlapping area on the solution accuracy and comparisons with the results from a dynamic mesh are also discussed.
\end{abstract}

Keywords: Numerical wave tank; Overset mesh; Floating body; Slamming

\section{Introduction}

In the past few decades, computational fluid dynamics (CFD) has increasingly been applied to aid the design and testing of new offshore and coastal structures. This is mainly due to the rapid development and advancement of both computing power and numerical algorithms for solving the underlying equations. Compared to the traditionally applied potential theory based solvers, high fidelity CFD simulations based on the full Navier-Stokes (N-S) equations can capture highly nonlinear free-surface effects and violent flow behaviours e.g. wave breaking and high order components of wave forces, which makes the N-S solution based free surface solvers in the form of a numerical wave tank (NWT) essential, as far as the evaluation of extreme wave loading on offshore structures and their survivability is concerned.

There have been a number of publications on the development and application of N-S solution based NWT. Park et al. (2003) developed a NWT based on finite difference schemes, in which a modified markerand-cell (MAC) algorithm was applied to track the free surface. Ong et al. (2017) applied a NWT to investigate free surface waves past two semi-submerged horizontal circular cylinders in tandem. The modelling framework was the open-source toolbox REEF3D, which is based on the finite difference method with a level-set free surface model. It has also been applied in e.g. Chella et al. (2015) for simulating wave breaking problems and Kamath et al. (2015) for calculating nonlinear wave forces on cylinders.

In the meantime, the open-source toolbox OpenFOAM ${ }^{\mathbb{R}}$ has gained popularity within the CFD community and has been adopted as the basis for constructing NWTs. The development of the Naval-Hydro

*Corresponding author, tel.: 44 (0)7802729519; e-mail address: cfd_hchen@outlook.com 
Package was such an example and it has since been applied to study various naval hydrodynamic problems e.g. green sea loads (Gatin et al., 2018). A solution decomposition model was further developed within the package in Vukčević et al. (2016), where only the perturbation components (equations) were solved as the incident field was readily available from potential flow theories. Other NWTs based on the OpenFOAM $^{\circledR}$ approach include waves2Foam (Jacobsen et al., 2012), IHFOAM (Higuera et al. 2013) and wsiFoam (Martínez Ferrer et al., 2016), which have become popular choices within the coastal and offshore research community for the analysis of e.g. ringing forces on a mono-pile (Paulsen et al., 2014), flow in and around oscillating water columns (OWCs) (Iturrioz et al., 2015) and moving paddle based wave generation strategy (Martínez-Ferrer et al., 2018).

In deep water conditions, offshore structures are usually floating on the free surface with a mooring system for station-keeping. Essentially, modelling such flow problems requires coupling between a free surface flow solver and a rigid body motion solver together with a proper adjustment to the mesh, in order to accommodate any moving/floating objects. For the latter, a moving or dynamic mesh method is an effective and popular option, as long as the motion amplitude is relatively small. Thus, the mesh can be moved/deformed in a stable and smooth manner during the calculation, see e.g. Palm et al. (2016) and Yu and $\mathrm{Li}(2013)$.

However, the moving mesh technique may significantly reduce the mesh quality, or even cause the simulation to diverge when the motion amplitudes become large. When rotation is the dominant motion, the arbitrary mesh interface (AMI) can be applied, where the sliding interfaces between grid blocks are employed. Otherwise, re-meshing of the local or even the entire grid may become necessary, where a new set of good quality meshes is generated and the data are transferred from the old mesh. Even with this approach, problems can still exist. Firstly, additional errors can be introduced during the interpolation of the data from the old mesh, which may already contain large errors in the solution due to the poor quality of the deformed mesh, to the new mesh. The problem can be further exacerbated for free surface flows, where sharp changes of fluid properties e.g. density and viscosity exist at the material interface (Bihs and Kamath, 2017). Secondly, frequent re-meshing can also have some adverse effects on the parallel efficiency of the underlying solver. Alternatively, an overset mesh approach can also be applied to model flow problems with moving objects. It is favourable to the moving mesh method owing to its ability to represent complex geometries whilst maintaining a good quality mesh, especially for large amplitude body motions. The overset mesh consists of sub-grids, which overlap one another; each one represents a different feature of the flow problem or separate body. This means that the features/bodies can be modelled independently of one another, and data can simply be exchanged between them. The method has long been developed for aerospace engineering applications (Chan, 2009). In recent years, it has also been adopted for solving some specific free surface problems (Ma et al. |2018).

Overset mesh functionality has been included in some of the commercial software, e.g. Star-CCM+ (Yang et al. 2018). However, it was not available in many of the major open-source toolboxes for a long time, which limited its usage by the researchers in the community. In the recent release of OpenFOAM ${ }^{\mathrm{R}}$ (ESI version v1706), an overset meshing functionality has been provided as an alternative to the existing dynamic mesh functionality for modelling moving objects. It has been applied in the recent works for modelling of some specific problems, e.g. Windt et al. (2018) and Paolo et al. (2018). In the present work, we are mainly concerned with a thorough evaluation of the new overset meshing functionality for modelling flow problems involving a free surface with moving/floating structures, which are widely encountered in many areas of the coastal and offshore engineering. This is achieved by integrating this functionality into an existing NWT based on OpenFOAM ${ }^{\circledR}$. It is hoped that the capability of the NWT can be further enhanced, and the NWT can be ultimately applied to model realistic free surface hydrodynamic problems involving highly nonlinear incident and scattered waves interacting with structures having complex geometries undergoing large amplitude motions.

The developed NWT has been validated against five cases covering a wide range of applications. The first three cases are 2D problems, where the main focus is to compare the results from the overset mesh and the moving/dynamic mesh, and check the sensitivity of the numerical solutions on the overlapping areas between the grids. Then the numerical model is further validated against two 3D cases, namely heave decay of a self-reacting floating point absorber and a lifeboat model in regular waves. Attention will be given on 
the performance e.g. accuracy and efficiency of the numerical model for modelling free surface flow problems with complex wave systems and geometry of structures.

\section{Description of the numerical model}

The present numerical model utilizes the well-known two-phase flow solver in OpenFOAM ${ }^{\circledR}$, namely the interFoam solver. Both air and water phases are considered in the numerical model, and the volume of fluid (VOF) method is used to track the free surface. Furthermore, to solve the floating body motion, a six degree of freedom motion solver is also adopted. The detailed mathematical formulations are given below.

\subsection{Governing equations}

\subsubsection{Free surface flow solver}

The governing equations in the fluid domain are the mass and momentum conservation for two incompressible, isothermal and immiscible fluids:

$$
\begin{gathered}
\nabla \cdot \boldsymbol{u}=0 \\
\frac{\partial \rho \boldsymbol{u}}{\partial t}+\nabla \cdot(\rho \boldsymbol{u}) \boldsymbol{u}-\nabla \cdot \mu \nabla \boldsymbol{u}=-\nabla p^{*}-(\boldsymbol{g} \cdot \boldsymbol{x}) \nabla \rho
\end{gathered}
$$

where $\boldsymbol{x}$ is the position vector, $\boldsymbol{u}$ is the velocity field, $\boldsymbol{g}$ is the gravitation, $\rho$ is the density field and $\mu$ is the viscosity field. $p^{*}$ is the excess pressure defined as:

$$
p^{*}=p-\rho \boldsymbol{g} \cdot \boldsymbol{x}
$$

where $p$ is the total pressure field. In order to capture the free surface, the VOF method is applied in the numerical model. Hereby a transport equation for the volume fraction field $\alpha$ is solved:

$$
\frac{\partial \alpha}{\partial t}+\nabla \cdot \boldsymbol{u} \alpha+\nabla \cdot\left(\boldsymbol{u}_{r} \alpha(1-\alpha)\right)=0
$$

Eq. (4) is a variant of the original transport equation, where the last term in the left-hand side is introduced. Here $\boldsymbol{u}_{r}$ is often referred as the compressive velocity field. In general, this term lacks physical meaning, since no relative motion between the water and air phase should exist in the VOF model at the free surface. However, it can efficiently prevent smearing of the interface whilst having a negligible effect on the accuracy of the solution, see Márquez Damián (2013).

\subsubsection{Rigid body motion solver}

The native rigid body motion solver in OpenFOAM, i.e. sixDoFRigidBodyMotion is applied to solve the six DoF (degree of freedom) motion for the floating body. The motion equation is formulated based on the linear and angular momentum conservations as:

$$
\begin{gathered}
\boldsymbol{a}_{f}=\boldsymbol{F}_{f} / m_{f} \\
\boldsymbol{\theta}_{f}=\boldsymbol{I}_{f}^{-1} \cdot \boldsymbol{M}_{f}
\end{gathered}
$$

where the subscript $f$ denotes the quantities for the floating body. Therefore, $\boldsymbol{a}_{f}$ and $\boldsymbol{\theta}_{f}$ are the linear and angular acceleration of the floating body, $m_{f}$ and $\boldsymbol{I}_{f}$ are the mass and moment of inertia of the floating body. $\boldsymbol{F}_{f}$ and $\boldsymbol{M}_{f}$ are the external force and moment, which are calculated as:

$$
\begin{gathered}
\boldsymbol{F}_{f}=\iint_{S}(p \boldsymbol{I}+\boldsymbol{\tau}) \cdot \mathrm{d} \boldsymbol{S}+\boldsymbol{F}_{\text {mooring }}+m_{f} \boldsymbol{g} \\
\boldsymbol{M}_{f}=\iint_{S} \boldsymbol{r}_{C S} \times(p \boldsymbol{I}+\boldsymbol{\tau}) \cdot \mathrm{d} \boldsymbol{S}+\boldsymbol{r}_{C M} \times \boldsymbol{F}_{\text {mooring }}+\boldsymbol{r}_{C G} \times m_{f} \boldsymbol{g}
\end{gathered}
$$



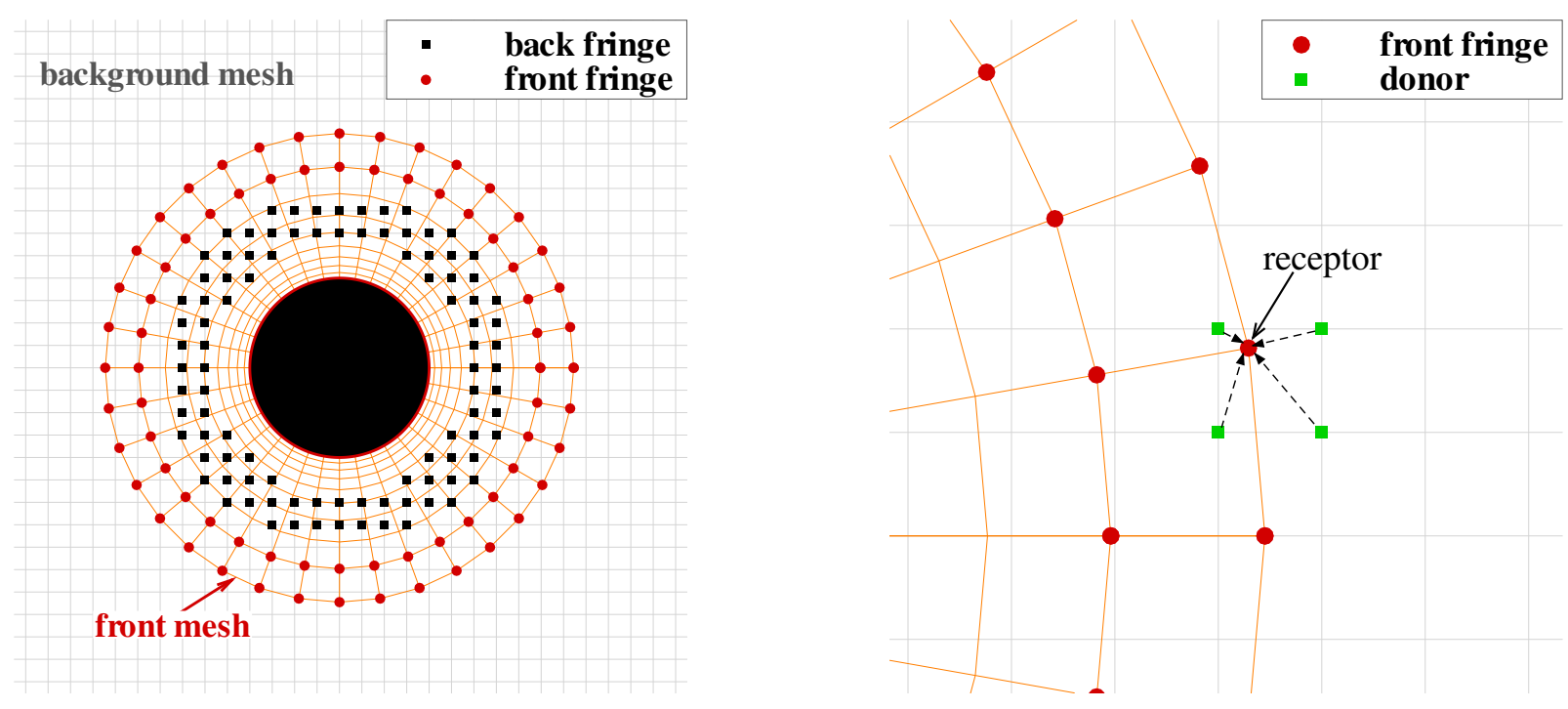

Figure 1: An example of an overset mesh for a 2D problem. The whole domain is overlayed by a background mesh and a single or multiple body-fitted component grids. Fringe cells/points are used to exchange information between different grids. Left: an overview of the domain; Right: a close view of a receptor and its donors.

where $\boldsymbol{I}$ is the identity matrix, $\boldsymbol{\tau}$ is the viscous stress and $S$ denotes the surface of the boundary patch for the floating body. $\boldsymbol{F}_{\text {mooring }}$ is the mooring force, while $\boldsymbol{r}_{C S}, \boldsymbol{r}_{C M}$ and $\boldsymbol{r}_{C G}$ are the arms of the hydrodynamic force, mooring force and gravity force, respectively. However, when the centre of mass and centre of rotation are identical, $\boldsymbol{r}_{C G}=\mathbf{0}$. Based on the acceleration from Eq. (5) and Eq. (6), the Newmark integration scheme is applied to obtain the velocity and position/orientation. For instance, the linear velocity $\boldsymbol{u}_{f}$ and position $\boldsymbol{x}_{f}$ are updated as:

$$
\begin{gathered}
\boldsymbol{u}_{f, n}^{k+1}=\boldsymbol{u}_{f, o}+\Delta t\left(\gamma \boldsymbol{a}_{f, n}^{k}+(1-\gamma) \boldsymbol{a}_{f, o}\right) \\
\boldsymbol{x}_{f, n}^{k+1}=\boldsymbol{x}_{f, o}+\boldsymbol{u}_{f, o} \Delta t+\beta(\Delta t)^{2} \boldsymbol{a}_{f, n}^{k}+(0.5-\beta)(\Delta t)^{2} \boldsymbol{a}_{f, o}
\end{gathered}
$$

where $\Delta t$ is the time step, $\gamma$ and $\beta$ are two parameters introduced in the Newmark scheme. We adopt a set of commonly used parameters with $\gamma=0.5$ and $\beta=0.25$, which yields the so-called constant average acceleration method. The second subscript for $\boldsymbol{x}_{f}$ and $\boldsymbol{u}_{f}$ indicates the value at either old time step (i.e. $\boldsymbol{u}_{f, o}$ ) or new time step (i.e. $\boldsymbol{u}_{f, n}$ ). Moreover, this scheme allows implicit sub-iterations within one time step, and the superscript $k$ indicates $\mathrm{k}$-th sub-iteration.

\subsection{Overset mesh}

The advantage of the overset mesh method is that it allows the use of several overlapping meshes to deal with complex integrated or separated geometries. Each component mesh can handle a part of the domain and has the freedom to move freely or as prescribed. A simple example of two overlapping meshes for a single cylinder is shown in Fig. 1. A background grid is firstly generated to discretise the whole domain without considering the geometry. Then a body-fitted grid dealing with the cylinder is generated on top of the background mesh. For complex problems, multiple component meshes can be generated and introduced into the whole domain. Before solving the flow equations, all the component meshes need to be assembled to determine the domain connectivity information. This includes several key steps: (1) hole cutting, (2) fringe search and (3) donor search.

- Hole cutting: Points/cells in (and close to) the objects, usually called hole points/cells, should be located and deactivated. As shown in Fig. 1, a part of background mesh close to the cylinder is 
blanked out. A common practice to "cut out" hole points/cells is to mark them with a tag i.e. $I B L A N K=1$ to indicate their exclusion from the the actual flow calculation.

- Fringe search: The points/cells surrounding the hole, usually named fringe points/cells, should be selected and used to exchange flow information between component meshes. As shown in Fig. 1, the black points on the background mesh and the red points on the front mesh are chosen as the fringe points.

- Donor search: For each fringe point/cell, one or several points/cells from a sibling mesh need to be selected as the donor(s) to send the information to the receptor. A demonstration is given in the right part of Fig. 1, which shows a receptor point on the front mesh and its donor points on the background mesh.

Algorithm 1 gives the detailed process of the overset mesh assembly implemented in the solver overInterDyMFoam. This solver utilizes a distance based implicit method aided by a regular voxel mesh to quickly cut out the hole from all the component meshes. A detailed description of this hole cutting method can be found in the works of Chan and Pandya (2015) and Druyor (2016). For fixed boundary problems, the assembly algorithm needs to be executed once only before the flow calculation. For deforming and/or moving boundaries, the assembly needs to be carried out for every time step.

Besides the overset mesh assembly, another crucial step of exchanging flow information between component meshes is needed to obtain the solution in the whole computational domain. This is accomplished through the use of fringes and donors. A fringe point/cell, also named receptor, receives solution information from its donors on sibling meshes. The flow variables $\phi_{r}$ at the receptor are obtained through an interpolation of the donors given by

$$
\phi_{r}=f\left(\phi_{1}, \phi_{2}, \ldots, \phi_{N}\right)
$$

in which $N$ is the number of donors. Linear, bi-linear, least square or other interpolation methods can be used to facilitate the exchange of information between component meshes. In the present model, we use a distance weighted function given by

$$
\phi_{r}=\frac{\sum_{i=1}^{N} \phi_{i} / d_{i}}{\sum_{i=1}^{N} 1 / d_{i}}
$$

where $d_{i}$ is the distance from a donor $i$ to its receptor. Given the interpolation scheme, the matrix can be assembled by correcting the off-diagonal elements as shown in Brunswig et al. (2010).

\subsection{Applied boundary conditions}

The governing equations are discretised by the finite volume method, making use of the IHFOAM toolbox developed by Higuera et al. (2013) for wave generation and active absorption. For all the simulations presented in Section 3, the tank bottom is treated as a slip boundary while no-slip condition is imposed on the floating body surface. Atmospheric condition is used on the top of the wave tank, where the velocity is set to the so-called pressureInletOutletVelocity, a native boundary condition supplied with OpenFOAM, which applies zero-gradient on all components of the velocity except where there is inflow, in which case a fixed-value condition is applied. The pressure boundary condition is set to totalPressure, indicating that the pressure is adjusted according to the velocity. For 3D problems, extra boundary conditions are needed on the side walls of the numerical wave tank. Again, slip condition is used, where the boundary layer development on the wall is safely neglected, since the floating body is sufficiently far away from the walls and it is unaffected by the local flow near the walls.

\subsection{Solution algorithm}

The overall flow chart is presented in Fig. 2. The so-called PIMPLE algorithm is used in the numerical model, which essentially is a combination of PISO (Pressure-Implicit with Splitting of Operators, Issa (1986)) and SIMPLE (Semi-Implicit Method for Pressure Linked Equations, Patankar and Spalding (1983)) algorithm. 


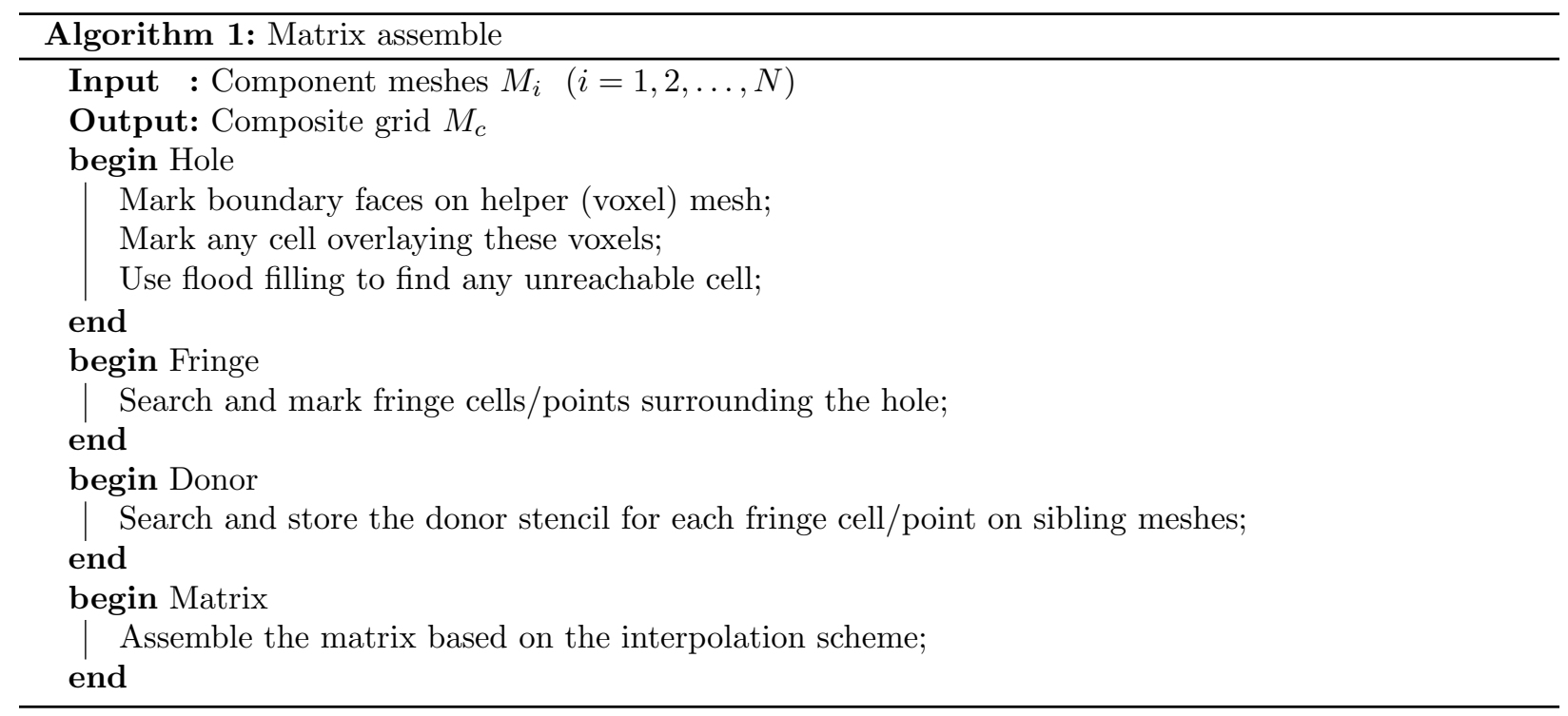

Recall that the algorithm is segregated to couple the free surface hydrodynamic solver and the rigid body motion solver. Hereby there exists a time lagging between the two solvers. At each time step, PIMPLE iterations are introduced in order to converge the numerical solution. Within each PIMPLE loop, the six DOF motion equation is solved first, with the update of the overset mesh. Then the VOF equation is solved to track the free surface. The velocity-pressure coupling is resolved by the PISO algorithm. However, the solution of momentum equation (momentum predictor) is optional, which is switched off in the present numerical model. Therefore, the velocity field from the previous time step is used for the following pressure correction steps. At the end of each loop, the numerical results are under-relaxed, which is naturally inherited from the SIMPLE algorithm.

\section{Validations and discussions}

In this section, we present a set of benchmark cases to validate our overset mesh based numerical wave tank. They typically cover a wide range of applications in the field of offshore engineering. It should be mentioned that no grid convergence study is presented in this work due to the limitation of pages. Convergence analysis has been done prior to the validations, where the background and body-fitted mesh were refined simultaneously and systematically. This ensured the grid similarity between each grid level. Adjustable time step was used to accelerate the computational speed with a Courant number limitation of $0.15-0.25$, which ensured a proper time resolution for wave generation and propagation in the NWT. The numerical solution was believed converged when the difference of the floating body motions from two consecutive grid levels was less than 5\%. All the cases presented below correspond to converged solutions.

\subsection{A 2D horizontal floating cylinder in regular waves}

The first validation case is on a $2 \mathrm{D}$ horizontal floating cylinder in regular waves. The original application of such a cylinder is on aquaculture fish farms, where the cylinder serves as a floater. In this section, the numerical model is set up based on both a dynamic mesh and an overset mesh, and their numerical results are compared.

\subsubsection{Experiments}

The experiments were conducted in a narrow wave flume at the Department of Marine Technology, Norwegian University of Science and Technology. Details on the experiments are presented in Kristiansen 


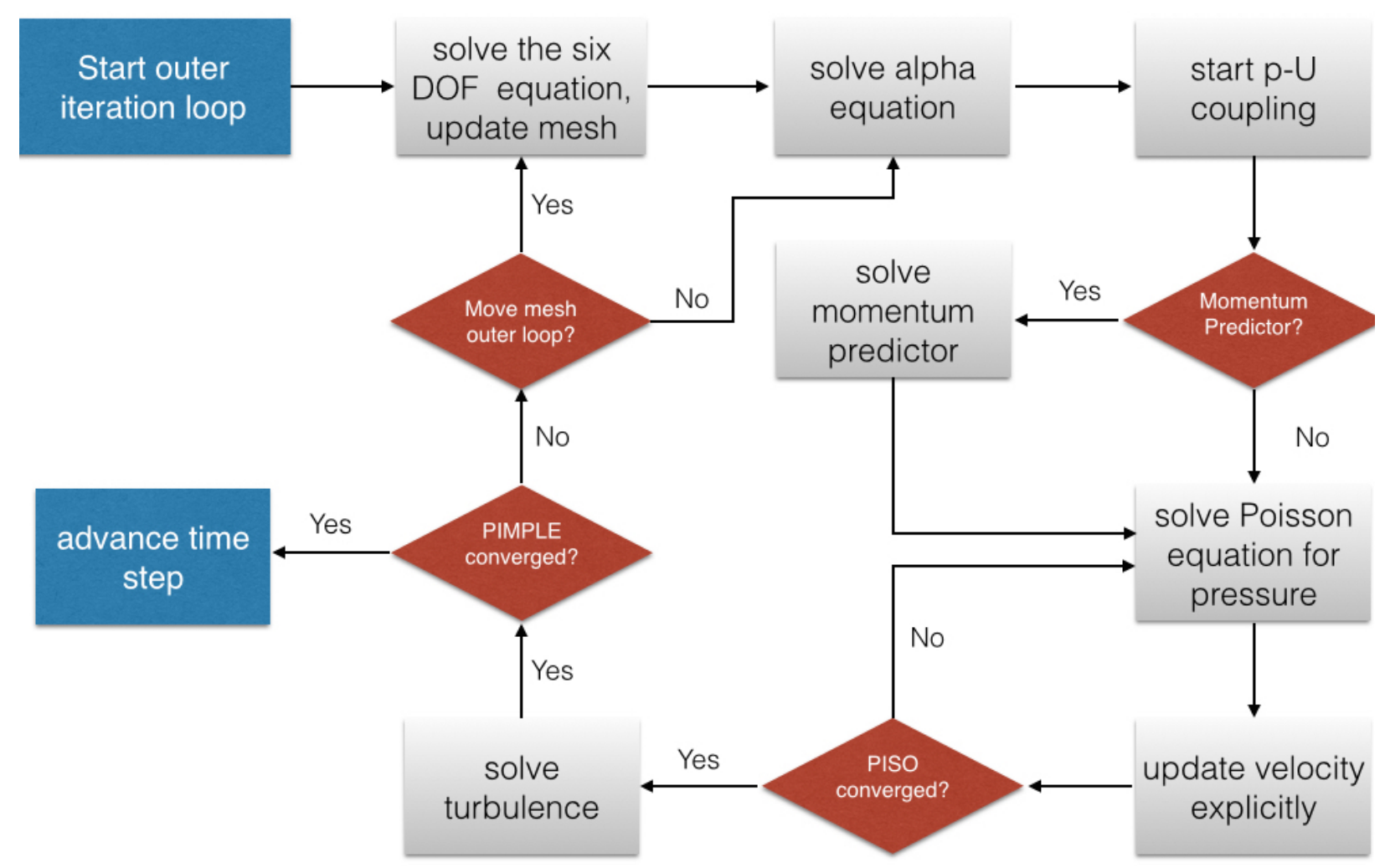

Figure 2: The flow chart of the segregated algorithm coupling the free surface flow solver and the rigid body motion solver.

Table 1: Selected incident wave conditions for the case with a horizontal floating cylinder in regular waves

\begin{tabular}{cccc}
\hline Case NO. & Wave height $H[\mathrm{~m}]$ & Wave period $T[\mathrm{~s}]$ & Wave steepness $[-]$ \\
\hline 1 & 0.086 & 0.878 & $1 / 14$ \\
2 & 0.120 & 1.038 & $1 / 14$ \\
\hline
\end{tabular}

(2010). The general setup of the experiments is given in Fig. 3. The flume had a dimension of $13.67 \times 0.6 \times 1.3$ $\mathrm{m}$ where the water depth was set to $1 \mathrm{~m}$. A horizontal cylinder was placed at the free surface whose mass was measured as $2.5 \mathrm{~kg}$. Two end-plates were equipped at two ends of the cylinder to reduce 3D effects. 5 $\mathrm{mm}$ gaps were left between the end-plates and the flume walls to avoid any contamination of experimental data due to contacts between them.

A single-flap wavemaker was equipped at the far left end of the tank, and a parabolic beach was put at the opposite end. The model was placed at a distance of $6.5 \mathrm{~m}$ from the wave-maker side and kept in position by mooring lines. Two mooring lines were applied on the wave-maker side of the model, while on the beach side, two lines that were attached to the model met to form a crowfoot.

A variety of incident wave conditions have been applied in the experiments. For validation purposes, two wave conditions are selected where the relevant parameters are given in Table 1. It can be seen that the wave steepness is the same for both cases. However, the absolute wave height is higher in case 2 .

\subsubsection{Setup of the numerical model}

A $2 \mathrm{D}$ numerical model is set up based on the experiments. The computational domain is shortened to be $5 \times 0.6 \times 1.5 \mathrm{~m}$. The water depth is set to be exactly the same as in the experiments, i.e. $1 \mathrm{~m}$. The cylinder is positioned $2 \mathrm{~m}$ downstream from the wave-maker. A linear restoring force is applied on each side of the cylinder to model the mooring systems. The end-plates are not modelled in the numerical model, since the 
(a)

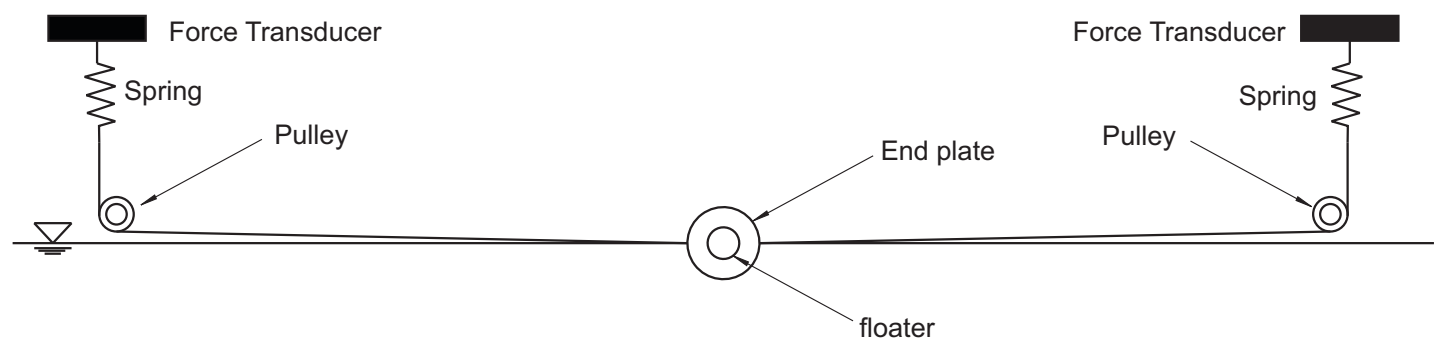

(b)

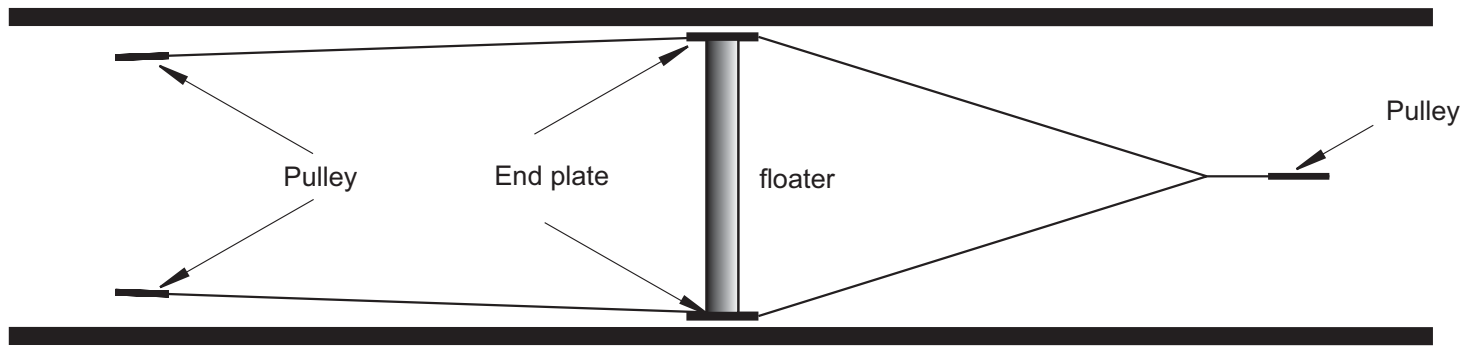

Figure 3: Setup of the experiments on a floating horizontal cylinder in regular waves: (a) side view. (b) top view. The cylinder was placed at the free surface, where end-plates with larger diameters were installed at two ends of the cylinder to reduce 3D effects. The flume had a dimension of $13.67 \times 0.6 \times 1.3 \mathrm{~m}$ and the water depth was set to $1 \mathrm{~m}$. On the wavemaker side, the cylinder was connected to two mooring lines which further connected to two pulleys and springs. On the other side towards the beach, two mooring lines were first joined before being connecting to the pulley and a single spring.

model is 2D. Instead, an equivalent mass is applied to account for its effect. The calculation procedure is also given in Kristiansen (2010). Essentially the mass of the cylinder is determined to be $3.940 \mathrm{~kg}$, which is used as an input in the numerical model.

Both an overset mesh and moving mesh are applied in the simulations. For the case with an overset mesh, a background layer of mesh is generated with regular hexahedral cells. The mesh is uniform in the horizontal direction. However, vertically it is refined at the free surface area. Essentially, this gives a mesh resolution of $\Delta x=0.016 \mathrm{~m}$ and $\Delta y=0.008 \mathrm{~m}$ at the free surface. Furthermore, another layer of body-fitted mesh is also generated near the cylinder. The domain for the body-fitted layer of mesh is O-type, whose diameter is four times the diameter of the cylinder. The mesh resolution in the body-fitted mesh is the same as the one at the free surface area in the background mesh.

Regarding the moving mesh, the resolution is quite similar to the overset mesh. In the far field, the mesh is the same as the background layer of the overset mesh. An O-type mesh is also applied in the vicinity of the cylinder, of which this area is exactly the same as the domain used in the body-fitted layer of the overset mesh. Fig. 4 depicts both mesh near the cylinder.

It should be clarified that for both cases, the computational domain, discretization schemes and the solution algorithms are exactly the same. However, the iterative solver for the linear system of the pressure equation is different. The reason is that for the overset mesh approach, the matrix from interpolation is asymmetric, while it is symmetric in the case with the moving mesh. Therefore, for the case with the moving mesh, the geometric-algebraic multi-grid (GAMG) with diagonal incomplete-Cholesky (DIC) preconditioner is used for the pressure equation. But in the case with overset mesh, the pressure equation is solved by a 


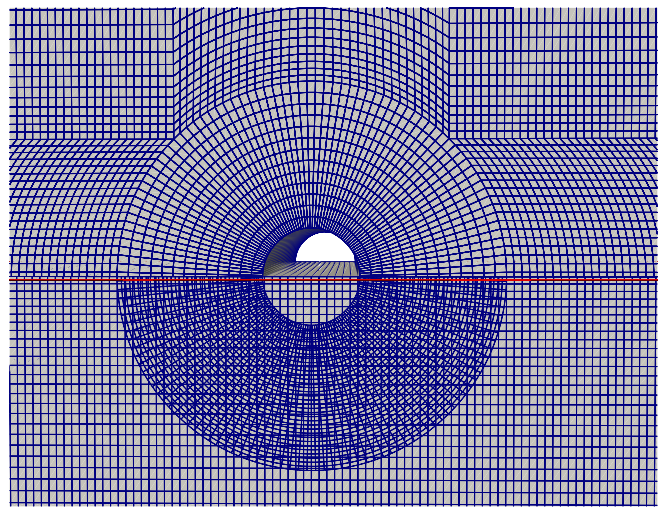

Figure 4: Comparison of the computational mesh near the cylinder used in both cases at $t=0$. The upper is the case using moving mesh technique. The lower is the overset mesh. The resolutions for both meshes are quite similar, and the areas with the O-type mesh are exactly the same.

stabilised preconditioned bi-conjugate gradient solver with the incomplete-LU preconditioner.

\subsubsection{Free surface flow}

Fig. 5 compares the hydrodynamic pressure field on the free surface from these two approaches. It is found that the results are very similar, but not exactly identical, especially near the cylinder. There exists slight difference during the run-up/overtopping and run-down process. This eventually leads to differences in the downstream waves. Fig. 6 further shows the local free surface and the mesh behaviour near the cylinder at different time instants. For the case with the moving mesh, the mesh during the simulation is deformed in order to conform with the cylinder boundary patch. Therefore, the grid velocity should be considered in the N-S equation when computing the flux for each variable. However, for the overset mesh, the body-fitted layer of mesh simply follows the motion of the cylinder. Then the variables are interpolated between both layers. Hence the different treatment of mesh is the main reason which results in the difference as shown in Fig. 5 and Fig. 6. In addition, the pressure solver may also influence the final results.

\subsubsection{Cylinder motion}

In this section we focus on the comparison of the cylinder motion using both dynamic mesh and overset mesh. The wave propagating in the empty wave tank is first compared against the experimental data, which is presented in Fig. 7. It should be mentioned that the generation method in the numerical wave tank and the physical laboratory is different. Moving paddle was applied in the experiments while the flux is directly injected from the boundary into the domain. Slight deviation is observed that the surface elevation in the numerical wave tank is smaller than from the experiments.

Fig. 8 and Fig. 9 present sway and heave motion from both cases under the wave conditions presented in Table 1. It is observed that the results are even more similar compared with the local pressure as shown in Fig. 5. This indicates that the local properties are more sensitive to the mesh type. Since the motion is an integral property, slight local change of pressure has rather limited effects on the motion of the cylinder. Compared with the laboratory tests, the agreement is satisfactory for both cases with both meshes. Minor differences are observed on the heave motion under both wave conditions. Strong nonlinearities are associated with such motions due to overtopping. The overtopped water generates negative pressure, which results in the double-peak shape of heave motion. This phenomenon is more pronounced in the numerical results.

\subsubsection{Comments on the computational time}

We also compare the efficiency of both mesh methods. With the current implementation of overset mesh, it is less efficient than the moving mesh technique. The computational time for the case with moving mesh technique is about $25 \%$ of the time for overset mesh. For moving mesh, the extra cost is on solving mesh 
$\mathrm{t}=4.5 \mathrm{~s}$
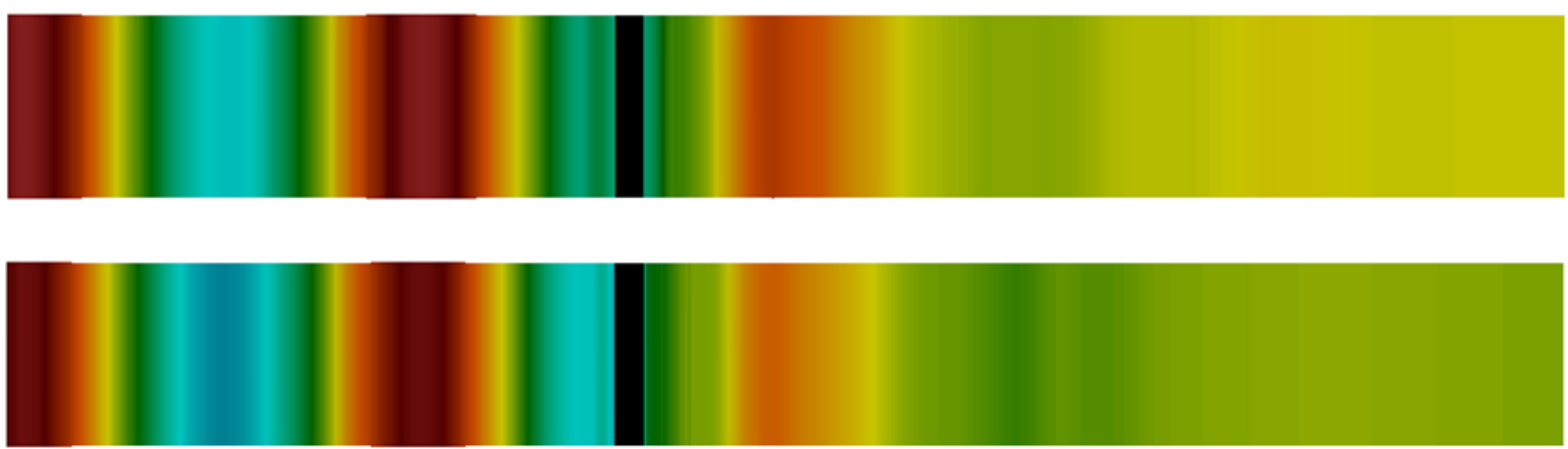

$\mathrm{t}=5.0 \mathrm{~s}$
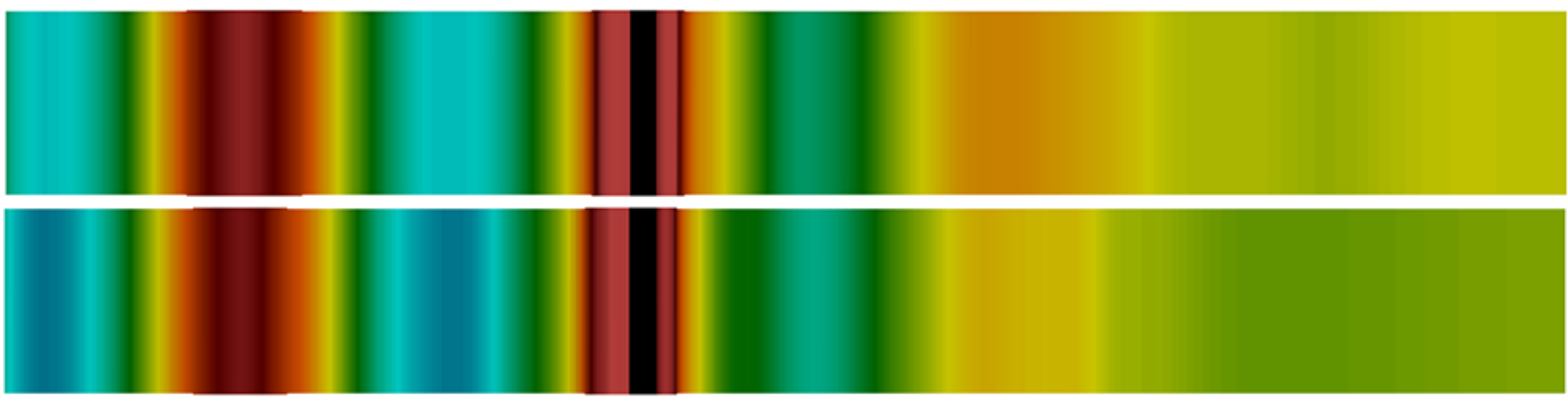

$\mathrm{t}=5.5 \mathrm{~s}$
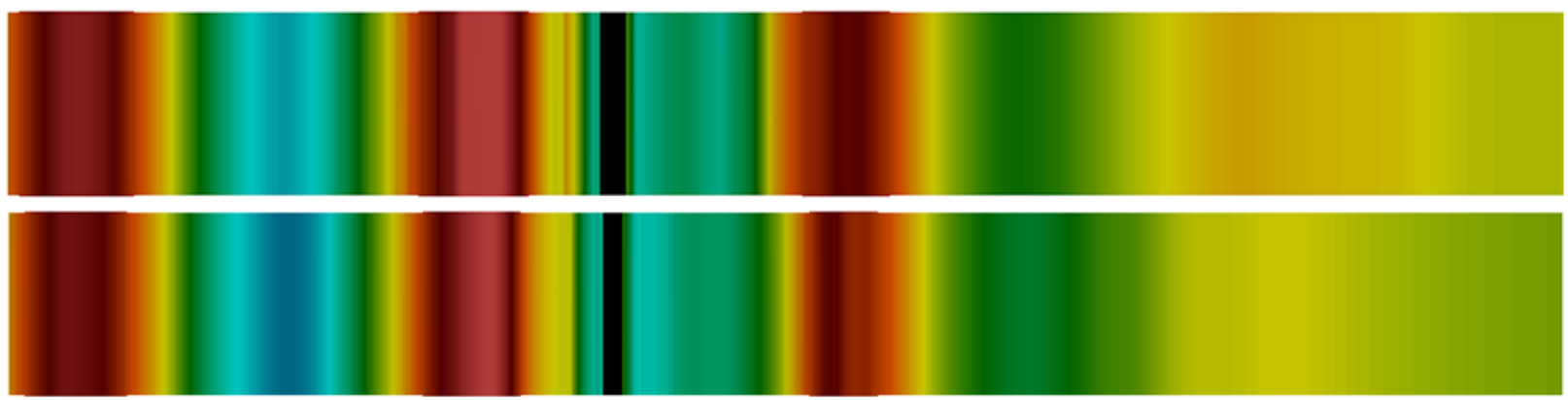

$\mathrm{p}$

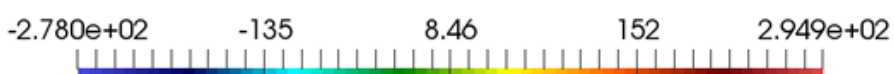

Figure 5: Comparison of the excess pressure on the free surface between the moving mesh (upper) and overset mesh (lower) approach for wave case 1 . The black strip indicates the position of the cylinder. 

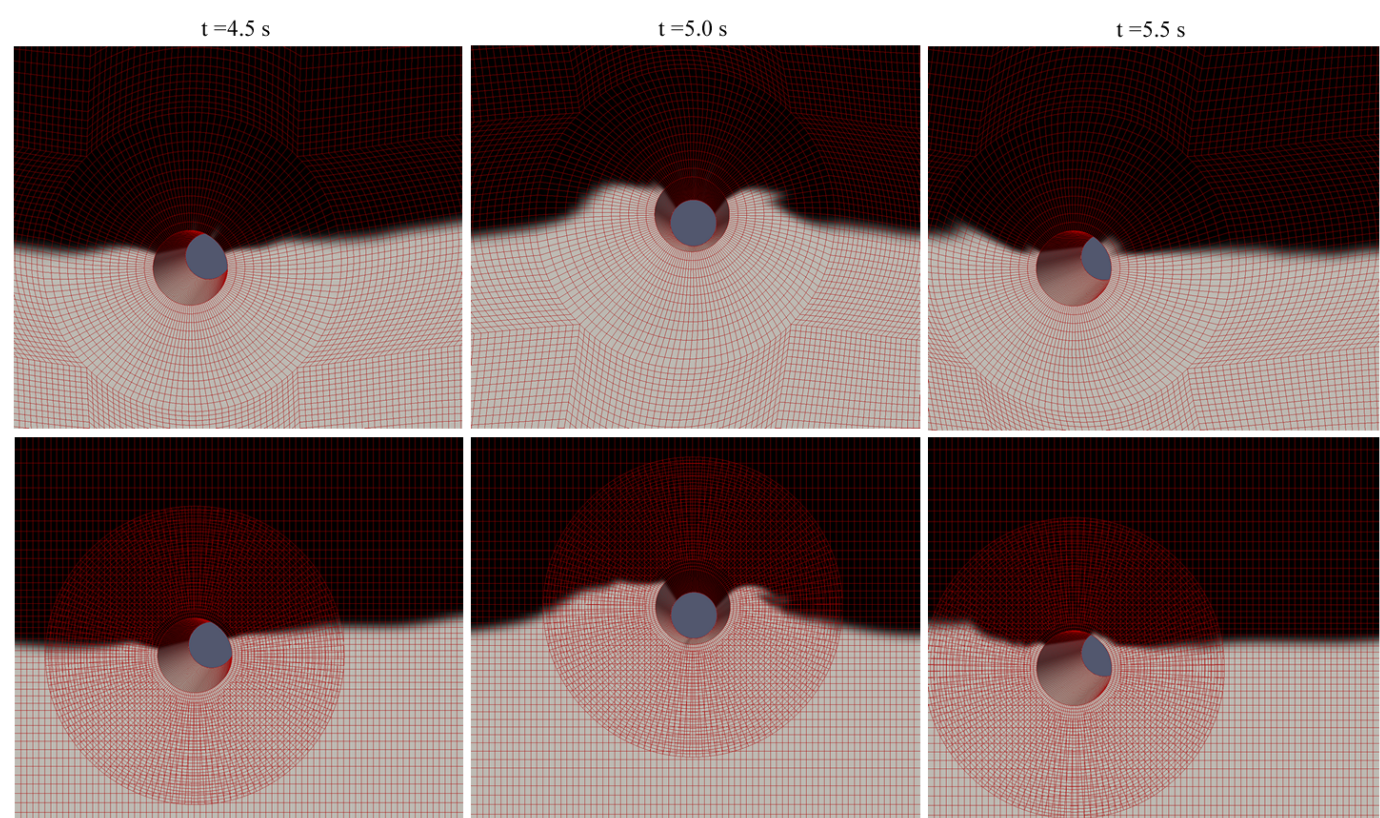

Figure 6: Comparison of the free surface details and the mesh near the cylinder for wave case 1. Upper: moving mesh. Lower: overset mesh. The white area indicates the water phase while the black area represents the air phase.

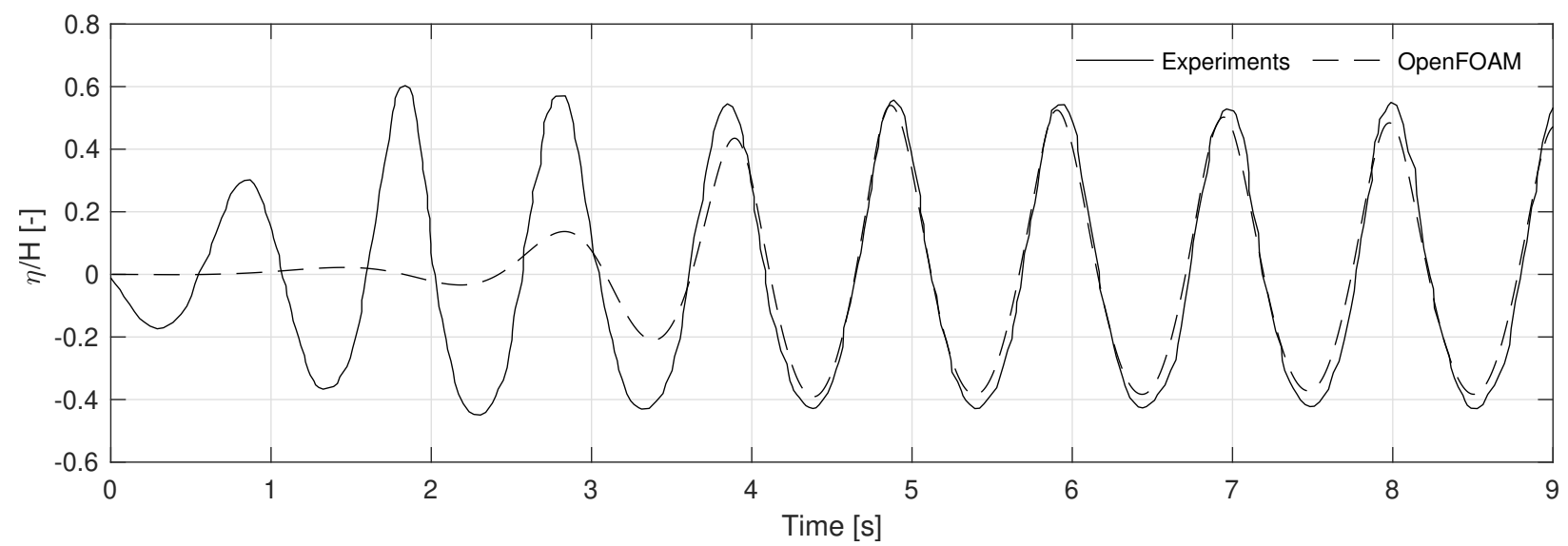

Figure 7: Comparison of the surface elevation for the case with wave propagating in the empty wave tank for Case 2. 

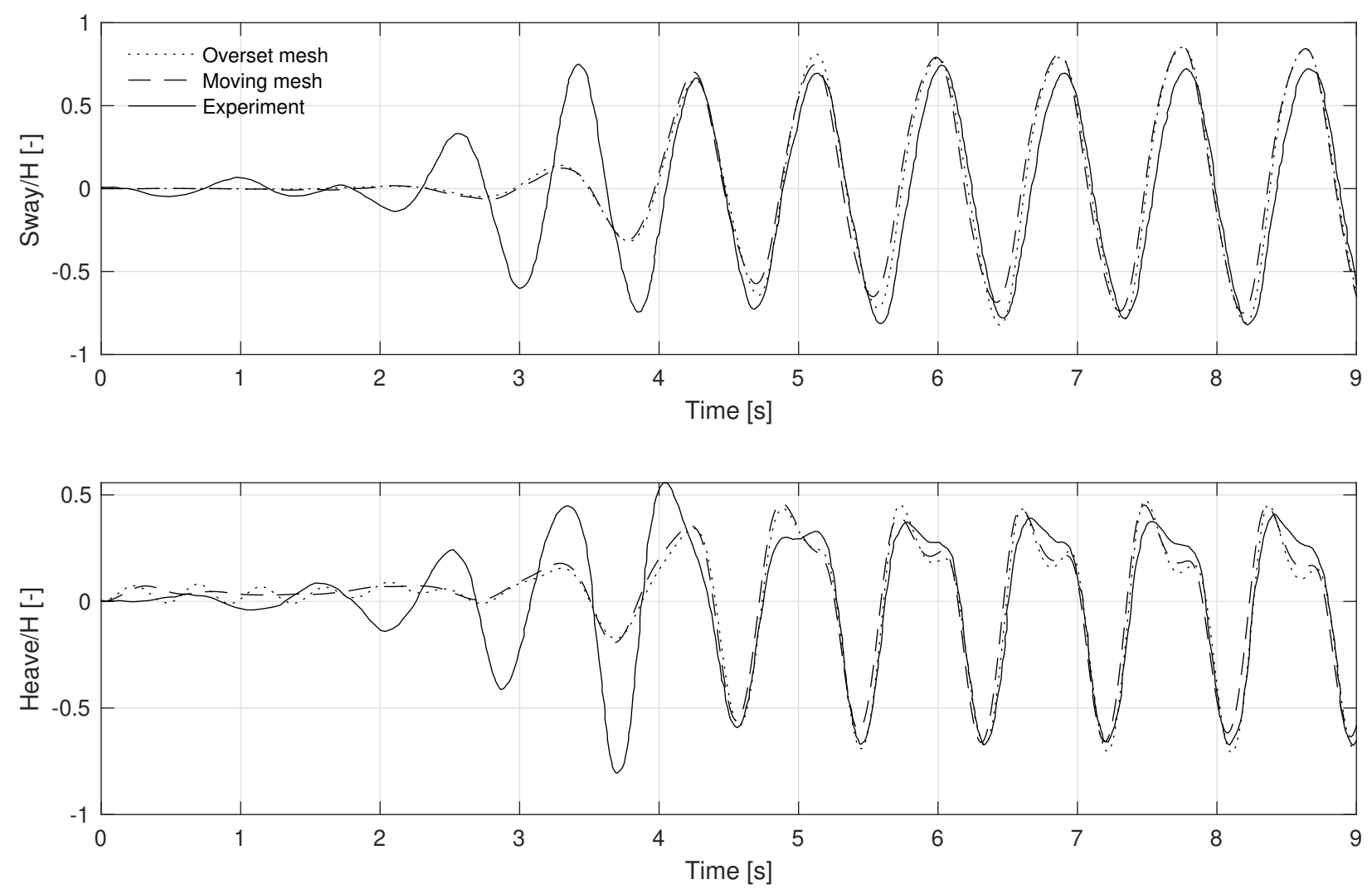

Figure 8: Comparison of sway and heave motion of the floating horizontal cylinder in regular waves between the experiments from Kristiansen (2010) and the numerical results from moving mesh and overset mesh. The incident wave condition is $\mathrm{T}=$ $0.878 \mathrm{~s}, \mathrm{H}=0.086 \mathrm{~m}$.

Table 2: The computational mesh, CPU number and CPU time in both cases. The CPU time is given as per wave period.

\begin{tabular}{ccccc}
\hline Mesh type & Mesh NO. [-] & CPU number [-] & CPU time (case 1/case2) [hour] & CPU Configuration \\
\hline Moving mesh & 68795 & 1 & $0.26 / 0.33$ & Intel Xeon E5-2600 \\
Overset mesh & 63025 & 1 & $1.04 / 1.30$ & Intel Xeon E5-2600 \\
\hline
\end{tabular}

motion equation, comparing with static mesh. However, the overset mesh needs to detect hole areas and interpolate the field values between two mesh layers at every time step, which essentially slow down the computational speed and reduce the efficiency. This has also been found in Windt et al. (2018).

\subsection{A $2 D$ box-shape floating object with a superstructure in regular waves}

This section further considers a 2D box-shaped floating object with a superstructure under a rather steep regular wave condition. The purpose is to examine the performance of the numerical model in terms of not only the floating body motion, but also the flow field around it. We also examine the sensitivity of the overlapping area on the accuracy of the numerical model.

\subsubsection{Experiments}

The experiments were conducted in a narrow wave flume at Research Institute for Applied Mechanics (RIAM), Kyushu University. The wave flume is $18 \mathrm{~m}$ long, $0.3 \mathrm{~m}$ wide and $0.7 \mathrm{~m}$ high. The water depth was set to be $0.4 \mathrm{~m}$. At the wave-maker side, it was equipped with a plunge-type wave generator. Meanwhile, at the other end, a wave-absorbing device was used. The incident wave condition was chosen to be $H=0.062$ $\mathrm{m}, T=1 \mathrm{~s}$. 

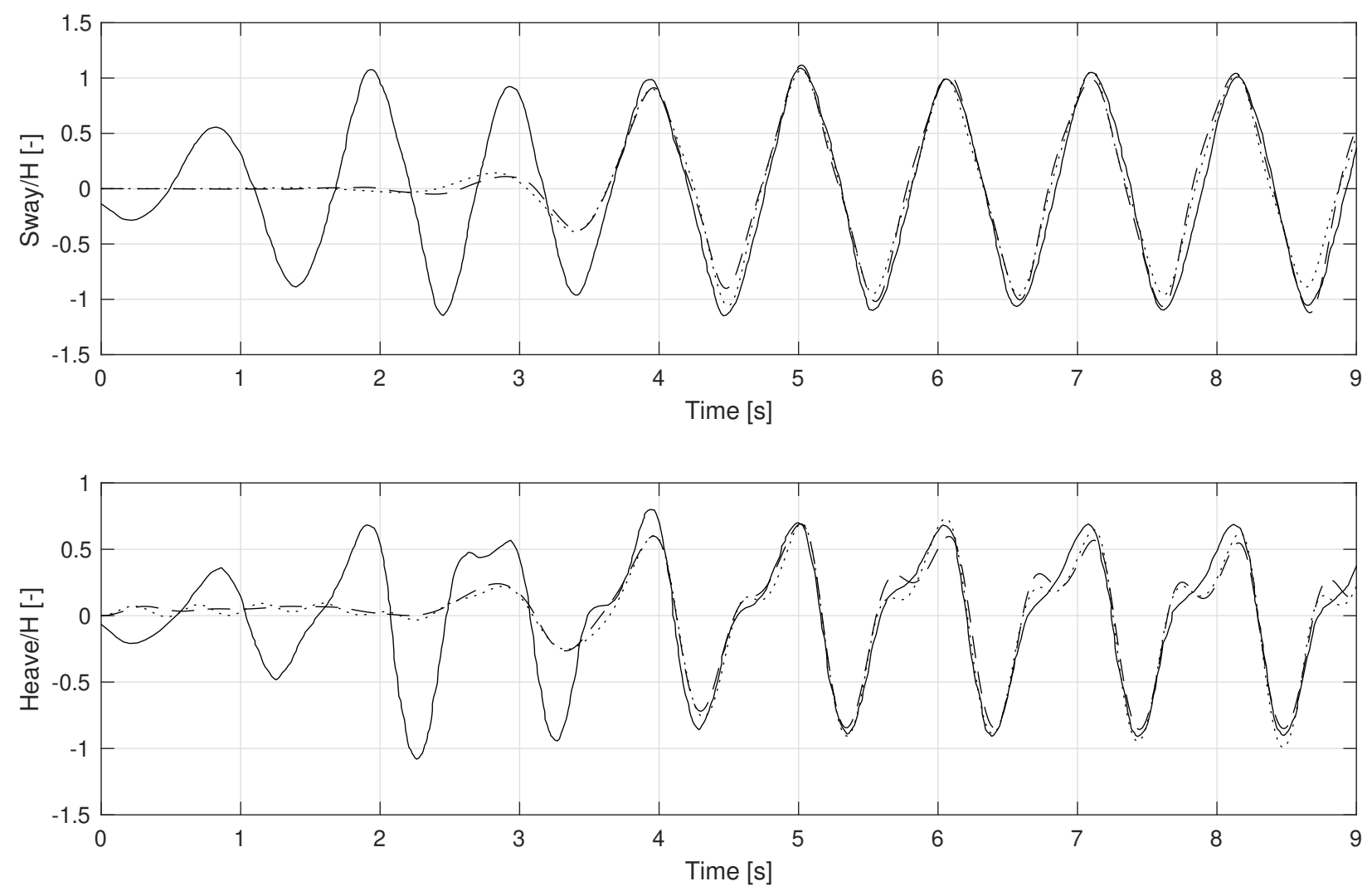

Figure 9: Same as Fig. 8 but for the case with $\mathrm{T}=1.038 \mathrm{~s}, \mathrm{H}=0.120 \mathrm{~m}$.

A box-shaped floating object was placed at $7 \mathrm{~m}$ from the wave-maker side. It was connected with a heaving rod through a rotational joint, and the heaving rod was set in between the slider mechanism installed in a carriage on guide rails. Therefore, it was allowed to heave and roll freely, but sway was restrained. A sketch on the setup of the experiments is given in Fig. 10, where the dimension of the floating body is presented. The mass of the body was measured to be $15 \mathrm{~kg}$ and roll moment of inertia was 0.3417 $\mathrm{kgm}^{2}$. Note that the centre of mass and the centre of rotation are not the same, since a rotational joint was installed to force the body roll around it. Readers are referred to Zhao and $\mathrm{Hu}(2012)$ for further details on the experiments.

\subsubsection{The computational domain and mesh}

A 2D numerical model is set up corresponding to the experiments. The dimension of the numerical wave tank is set to be $8.3 \times 0.3 \times 0.973 \mathrm{~m}$. In the background mesh, the domain is discretised uniformly by 0.3 million cells, which gives a cell size of $0.005 \times 0.005 \mathrm{~m}$. Three different domain sizes are tested for the overlapping area. The dimensions of these are $0.7 \times 0.6 \mathrm{~m}, 1.0 \times 0.6 \mathrm{~m}$ and $1.3 \times 0.6 \mathrm{~m}$, respectively. A snapshot of the outlines of different overlapping domains is presented in Fig. 11. In all the three cases, the resolution of the body-fitted mesh is the same as the background mesh.

\subsubsection{Computational results}

Fig. 12 presents both the numerical and experimental results for the flow field near the floating boxshaped body within one wave period. It is found that quite similar flow fields are generated by the numerical model with different overlapping areas. Therefore, the overlapping domain size actually has a negligible effect on the flow field. Only some flow details such as small surface ripples are not identical. Compared with the experimental results, it is concluded that the numerical model can produce a reasonable flow field around 


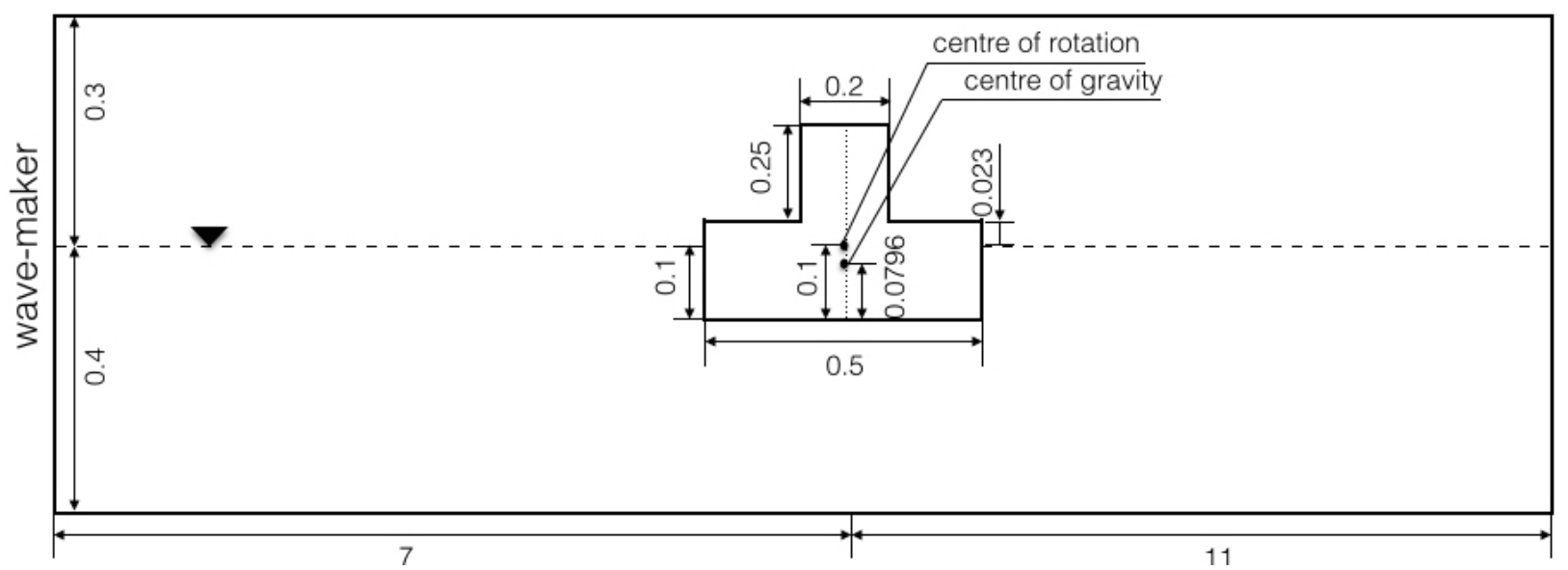

Figure 10: Sketch of the general setup of the experiment for the floating box-shaped body with superstructure in waves (unit: $\mathrm{m})$. The flume has a dimension of $18 \times 0.3 \times 0.7 \mathrm{~m}$. The still water depth is set to $0.4 \mathrm{~m}$, and the box-shaped body is placed 7 $\mathrm{m}$ downstream from the wave-maker side.

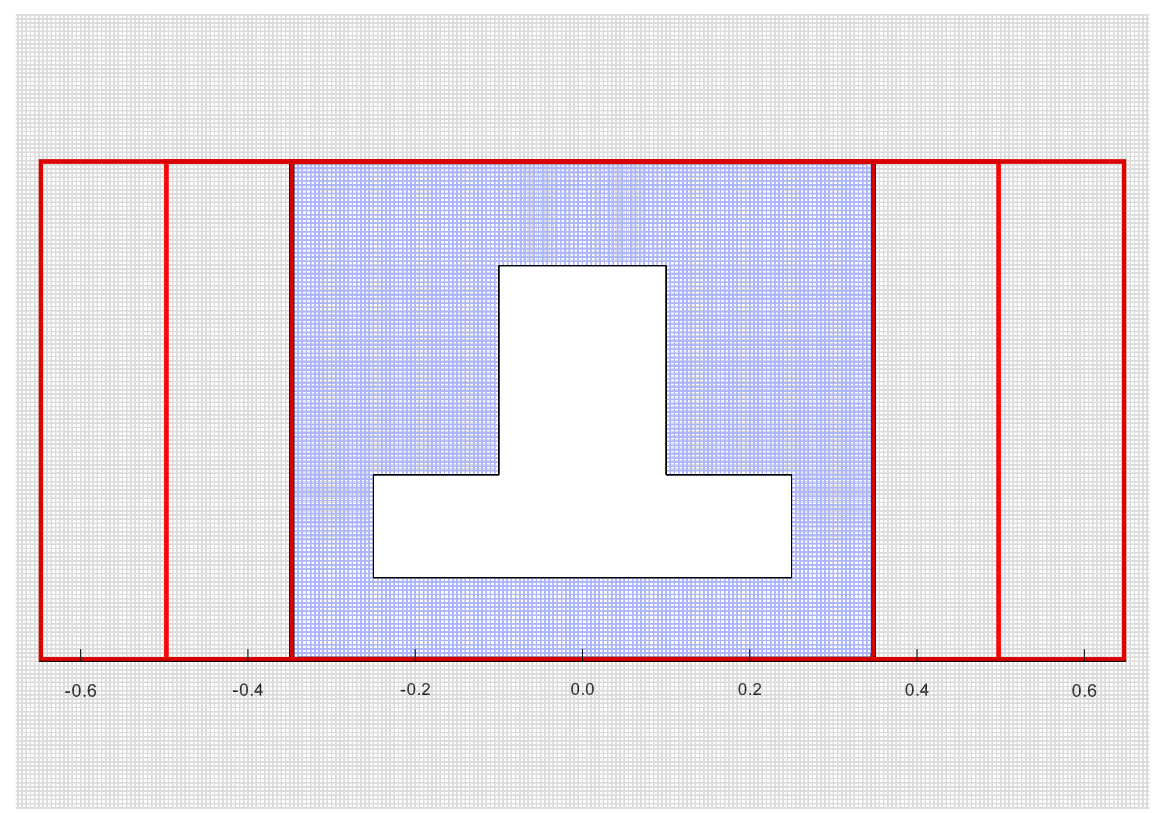

Figure 11: Three different overlapping areas and the corresponding mesh used in the numerical simulations for wave interaction with floating box-shaped body with superstructure. Note that the mesh resolutions in all the three cases are the same. 
the floating body including the wave run-up and run-down processes. However, the volume of water on deck seems to be underestimated, which is shown in e.g. $t=6.9 \mathrm{~s}$.

The heave and roll of the floating body and the surface elevation at $1.9 \mathrm{~m}$ in front of the body are presented in Fig. 13 for both numerical and experimental results. The numerical results with different overlapping areas collapse into one line, which again confirms our previous conclusion that the overlapping domain size has negligible effect on the computational results. Regarding the accuracy of the numerical results, the heave motion and the surface elevation in front of the body are well predicted. However, the trough value of roll motion is underestimated. This indicates that the amplitude of the roll motion in the counter-clockwise direction is underestimated, probably due to the underestimation of water on deck, where less negative pressure is generated. Therefore, the numerical model actually produces an asymmetric roll motion.

\subsubsection{Comment on the selection of overlapping area}

As we notice from the above results, the overall effects of overlapping domain size on the computational results are trivial. However, the computational costs for the three cases are different, where the case with larger overlapping area is more time-consuming. This is due to the difference in the cost for solving the equations on the overlapping domain. Practically, it is suggested that the overlapping area should be reasonably small, in order to reduce the computational cost. However, the boundary of overlapping domain should be sufficiently far away from the position where any sharp gradient occurs, for instance near a boundary layer. This may result in a discontinuity in the solution due to interpolation between both layers.

\subsection{Water entry problem}

Ship slamming is an important topic within the field of naval hydrodynamics. It is a violent short duration impact between the fluid surface and the hull, which typically occurs in rough weather with steep waves. In the present study, we consider two $2 \mathrm{D}$ cases, i.e. the wedge-shape body with constant speed and free-fall of a ship-like section.

\subsubsection{A 2D wedge-shaped body impact with constant speed}

In previous experimental and numerical studies, 2D wedges have been commonly used in the study of impact loads due to its simple and well-defined geometry. Fig. 14 shows a general configuration for the present wedge impact problem. The domain is set to be a square shape with a side length of $16 \mathrm{~m}$. Initially a wedge is held still with its tip on the free surface. Then it is driven into the water with a constant velocity $w_{0}=1 \mathrm{~m} / \mathrm{s}$. In the present work, two wedges with different deadrise angles are tested, one with $\phi=30^{\circ}$ and the other with $\phi=45^{\circ}$ (see Fig. 14 for definition of deadrise angle $\phi$ ). The length for top side of both wedges is $2.4 \mathrm{~m}$. The overset mesh based model is set up where a region of $[-2.4 \mathrm{~m}, 2.4 \mathrm{~m}] \times[-2.4 \mathrm{~m}, 3.2$ $\mathrm{m}$ ] enclosing the structure is chosen to generate the body-fitted layer of mesh. Fig. 15 presents the mesh topology used in the simulation. The mesh is refined at the free surface area horizontally and at the tip vertically. The ensures a good resolution where violent flow occurs.

The pressure coefficient $C_{p}$ is presented in Fig. 16 for both cases at three time instants, where $C_{p}$ is defined as $C_{p}=\left(p-p_{0}\right) / 0.5 \rho v^{2}$. Meanwhile, Fig. 16 also gives the similarity solution from Dobrovol'Skaya (1969) and the analytical solution from Mei et al. (1999). It can be seen that the dimensionless form of the results at different times is very similar to each other, since they are self-similar in a short period, during which inertia effect is dominant over gravity. For the case with $\phi=30^{\circ}$, the present model coincides better with the similarity solution. Meanwhile, regarding the results with $45^{\circ}$ wedge, when $z / v t<0$, the present model agrees better with the analytical solution. But for the part of $z / v t>0$, the results start getting closer to the similarity solution. In addition, the free surface profile is depicted in Fig. 17. Again, the dimensionless form of the surface profiles is self-similar to each other within a short impact period for both wedges, where a reasonable thin layer of jet-type flow along the side of the wedge is generated. 


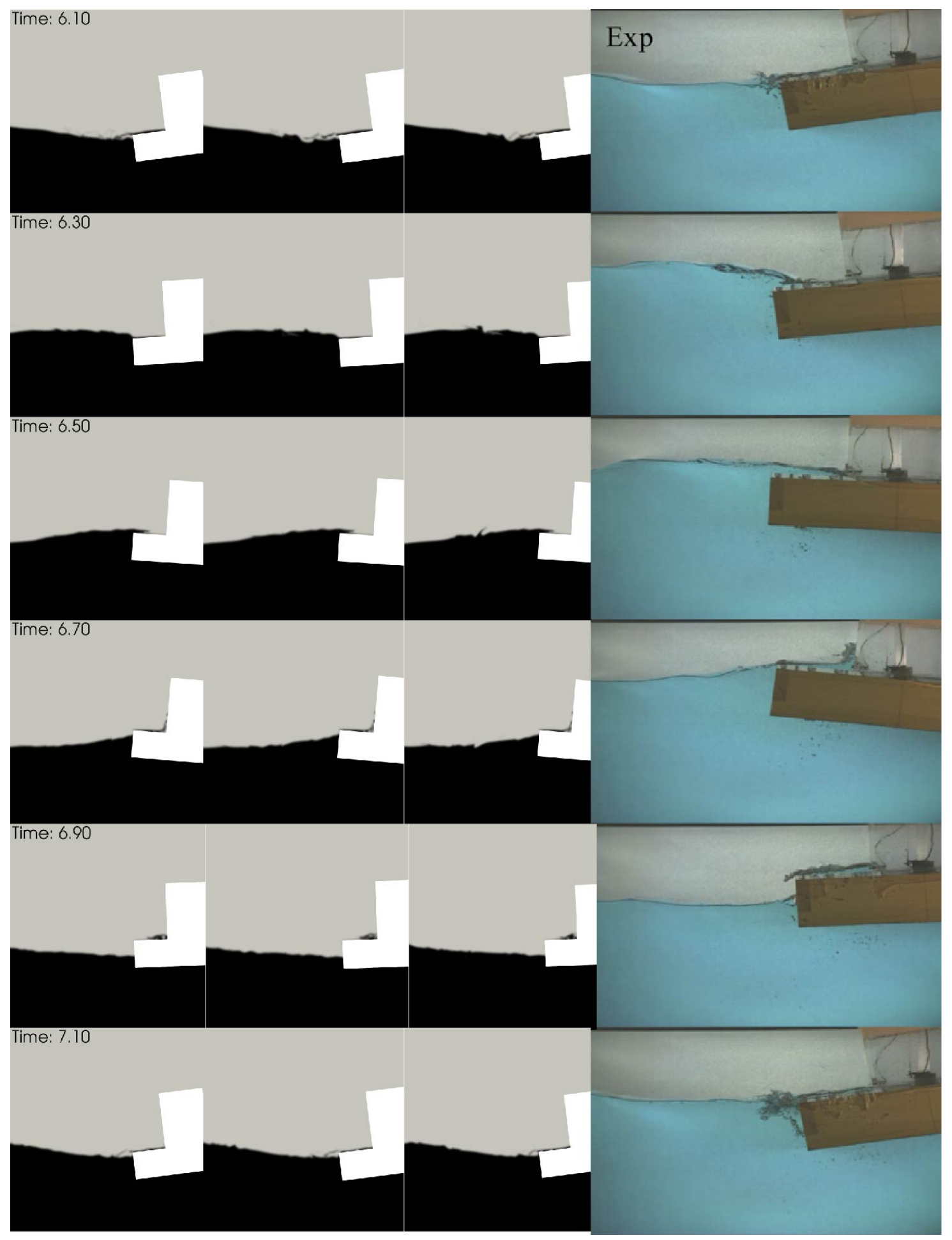

Figure 12: Comparison of the free surface and body positions between the numerical simulations with different overlapping domain sizes and the experimental results within one wave period. The first three columns from left to right are simulation results with small, medium and large overlapping domains. The most right column of figures are the reproduction of experiemntal results from Zhao and $\mathrm{Hu}(2012)$. 

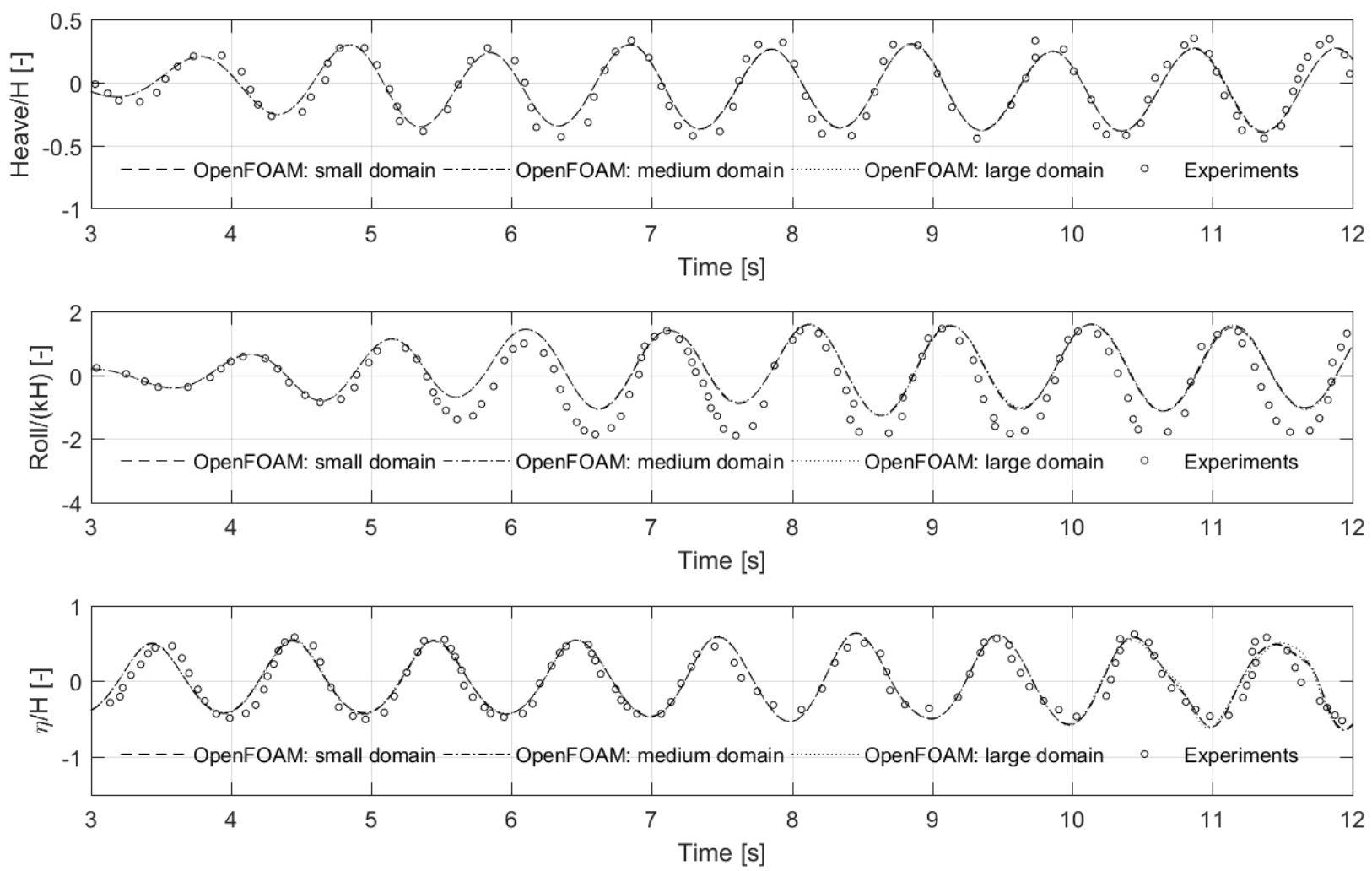

Figure 13: Comparison of the heave and roll motion of the box-shaped floating body with superstructures, and the surface elevation at $1.9 \mathrm{~m}$ in front of the body between the numerical results from the present model with three different overlapping domain sizes and the experimental data from Zhao and $\mathrm{Hu}(2012)$. The heave, roll and surface elevation were non-dimensionalized where $k$ is the wave number and $H$ is the wave height.

\subsubsection{Free fall of a ship-like section}

In 2016, the International Society of Offshore and Polar Engineers (ISOPE) organised a collaborative experimental and numerical study of ship slamming problems, where the Korea Research Institute of Ships \& Ocean Engineering conducted experiments on the free fall of a $2 \mathrm{D}$ ship-like section. In this section we numerically reproduce the experiments. The geometry of the section is given in Fig. 18, where a pressure sensor is installed on the side of the body to measure the local pressure. Initially the section is held still where the tip is just on the free surface. Then it is released to fall freely into the water. Since in this case the dropping height is zero, dynamic mesh is also feasible for modelling the entry process. Hereby both methods are used for the purpose of comparison, where their grid resolutions are in general quite similar. However, the domain size is different. With overset mesh method, the computational domain is $2 \times 2 \mathrm{~m}$, while it is $4 \times 4 \mathrm{~m}$ with dynamic mesh. The reason is that a relatively large domain can properly reduce the distortion of the mesh near the moving boundary when using dynamic mesh, which benefits the stability of the numerical solution.

Fig. 19 presents a comparison of the local pressure at Point P (see Fig. 18), as well as the displacement, the velocity and acceleration of the section from the present overset mesh based model, the dynamic mesh model and the experiments. In general, the kinematics of the section are well predicted by both numerical models, except from $t=0.3 \mathrm{~s}$ and afterwards where the velocity is overpredicted by both models. This may be partially due to the use of a spring in the laboratory test to prevent the section from impacting the bottom of the tank. However, the hydrodynamic pressure is not captured so accurately as the kinematics of the wedge, which is overestimated by both models. The overset mesh based model produces a relatively better result, which has less discrepancy with the experimental data. Actually this peak is formed as a 

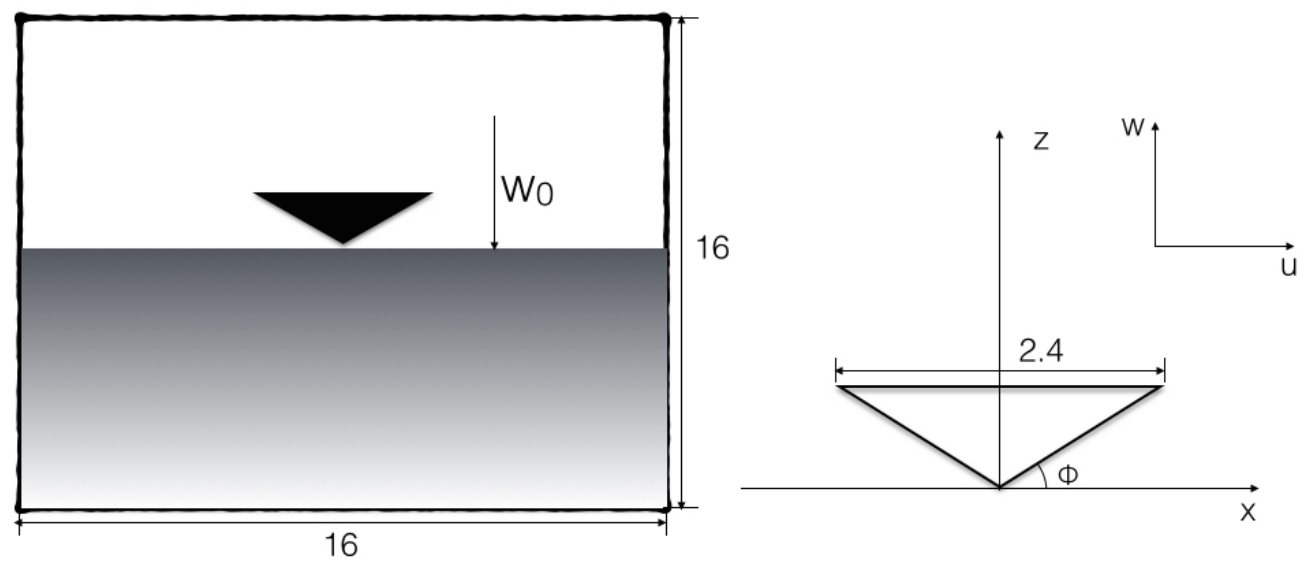

Figure 14: A sketch on the setup of the numerical model for 2D wedge shaped body impact problem (unit: $m$ ). Left: an overview of the setup of the numerical model. Right: close-up view of the $2 \mathrm{D}$ wedge shaped body, and definition of the deadrise angle $\phi$.
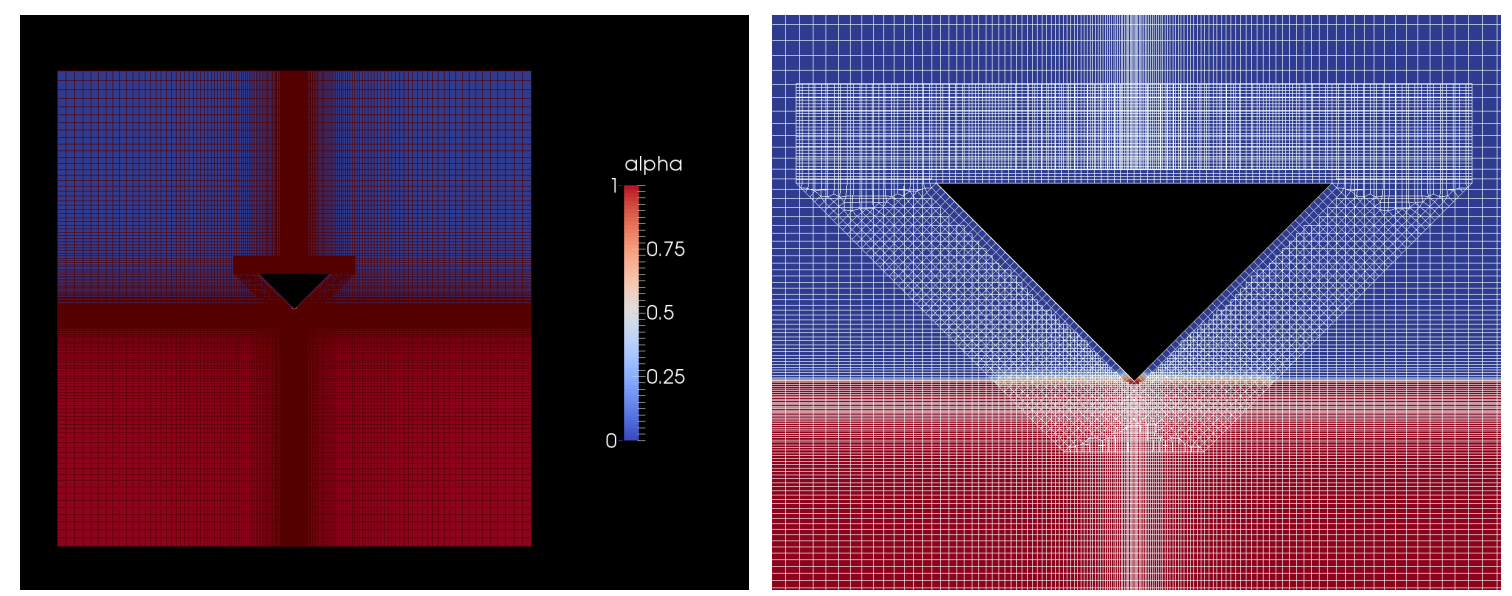

Figure 15: The overset mesh used in the numerical simulation for 2D wedge impact problem $\left(\phi=45^{\circ}\right)$. Left: An overview of the mesh topology. Right: a close-up view at the overlapping area around the wedge.

result of the evolution of the free surface, which has been shown in Fig. 20. Approximately from $t=0.25 \mathrm{~s}$, the free surface reaches the location of the pressure sensor, and the relative velocity between the free surface and the body induces the peak in the pressure. With the increase of the entry level, the pressure rapidly drops. Meanwhile, due to the curvature of the section near the tip, a thin layer of air is entrained along the side of the wedge. Finally at $t=0.39 \mathrm{~s}$, the section keeps dropping where its top side is below the free surface.

\subsection{Free decay test of a locked self-reacting floating point absorber}

A self-reacting floating point absorber (SRFPA) is a kind of wave energy converter (WEC) that has been widely used all over the world. It is operated predominantly in heave motion, and extracts energy from the relative motion between the floating and reacting plate. The energy capture of the device reaches the maximum when the system resonates with the input waves. However, under extreme wave conditions, the SRFPA is automatically locked to increase survivability, so no relative motion occurs between the floater and the reacting plate. In this section we conduct a numerical simulation on the heave free decay test of the locked SRFPA. The results are compared with the experimental data and a BEM (boundary element method) result. 

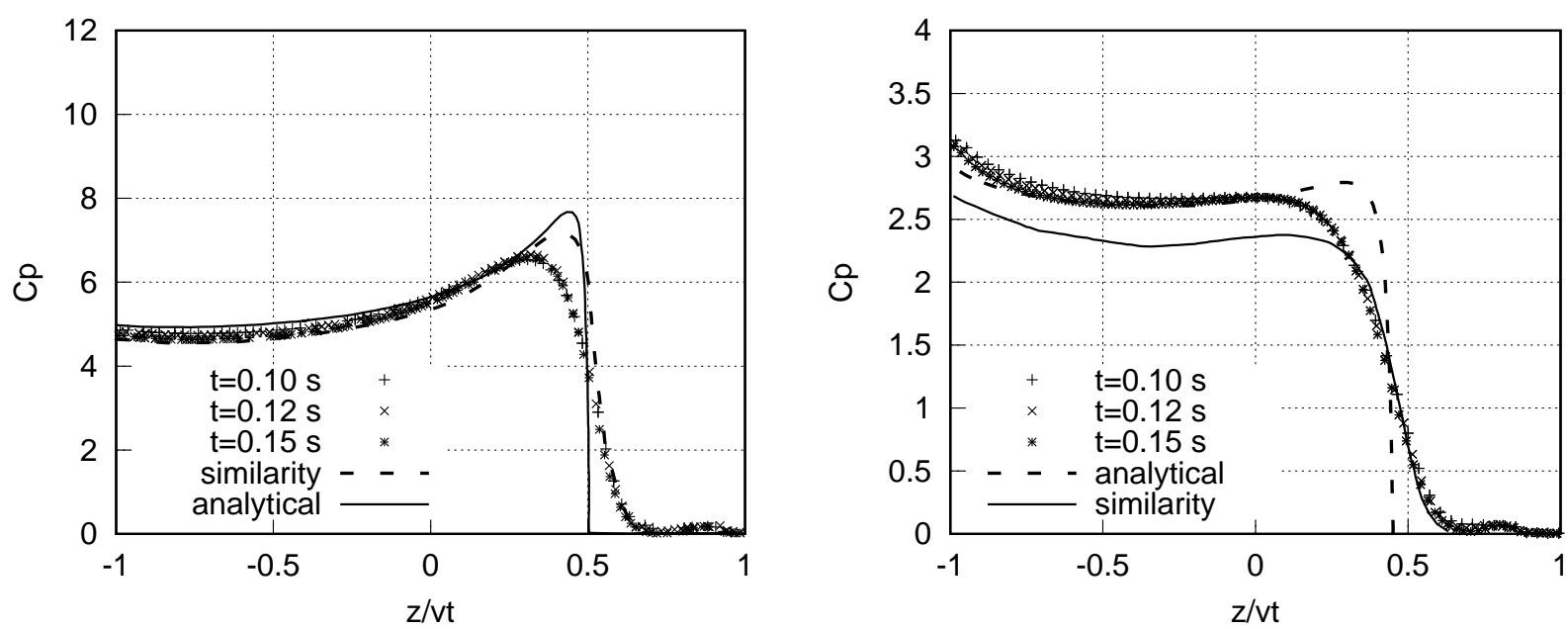

Figure 16: Pressure coefficient $C_{p}$ (defined as $\left.\left(p-p_{0}\right) / 0.5 \rho w^{2}\right)$ on the wedges from the present model, the similarity solution from Dobrovol'Skaya (1969), the analytical solution from Mei et al. (1999). Left: $\phi=30^{\circ}$. Right: $\phi=45^{\circ}$.

\subsubsection{Experiment}

The experiment was performed in December 2010 at UC Berkeley's wave tank, which is $68 \mathrm{~m}$ long 2.4 $\mathrm{m}$ wide and $1.5 \mathrm{~m}$ deep. A model-scale SRFPA was built based on the SolidWorks design as shown in Fig. 21(a), which includes all the components of the SRFPA structure. A 2D motion tracking system was utilized to capture the FPA motion, and the system used passive markers on the buoy to create targets for the motion tracking software. Eventually with the weight of the load cell and the tracking target, the total mass of the 1/100-scale FPA model in the experimental test was $313 \mathrm{~g}$. It was initially lifted from the equilibrium position by $0.02 \mathrm{~m}$. Then it was freely decayed in heave motion. More details on the experiment can be found in $\mathrm{Yu}$ and $\mathrm{Li}(2013)$.

\subsubsection{Setup of the numerical model}

A square shape wave tank is used in the numerical model, since the radiated waves propagated in all directions. The side length of the domain is set to $2.4 \mathrm{~m}$ while the depth is the same as in the experiment, i.e. $1.5 \mathrm{~m}$. Furthermore, only a basic structural design of the FPA is considered in the model, where the supporting jacket and the details of the reaction plate are neglected, as shown in Fig. 21(b). In order to absorb the radiated waves, an active wave absorption boundary condition in IHFOAM is applied on all the side walls. This can effectively absorb the radiated waves in case it influences the decay of the FPA. Meanwhile, the bottom is set to a slip wall since it has negligible effect on the decay motion.

A snopshot of the computational mesh is given in Fig. 22. Regarding the background mesh, in the longitudinal and transverse direction, the mesh is refined towards the centre lines respectively. Furthermore, in the vertical direction, it is refined near the free-surface and the body. The body-fitted mesh is generated using the snappyHexMesh utility. The geometry of the FPA is represented by a stereo lithography surface file. Then the mesh gradually conformed to the surface by iteratively refining a starting mesh and morphing the resulting split-hex mesh to the surface. This finally produces a total number of 7.5 million cells for the model including both the background and the body-fitted mesh.

\subsubsection{Radiated waves}

Fig. 23 shows the radiated waves due to decay of the FPA. Nearly circular wave fronts are observed due to symmetric shape of the body. With the oscillation of the FPA, energy is transfered from the FPA to the radiated waves, which serves as an important source for the damping of FPA, i.e. potential damping. Eventually when the radiated waves reach the side walls, they are absorbed by the active absorption boundary condition. No standing waves due to reflection are observed. 

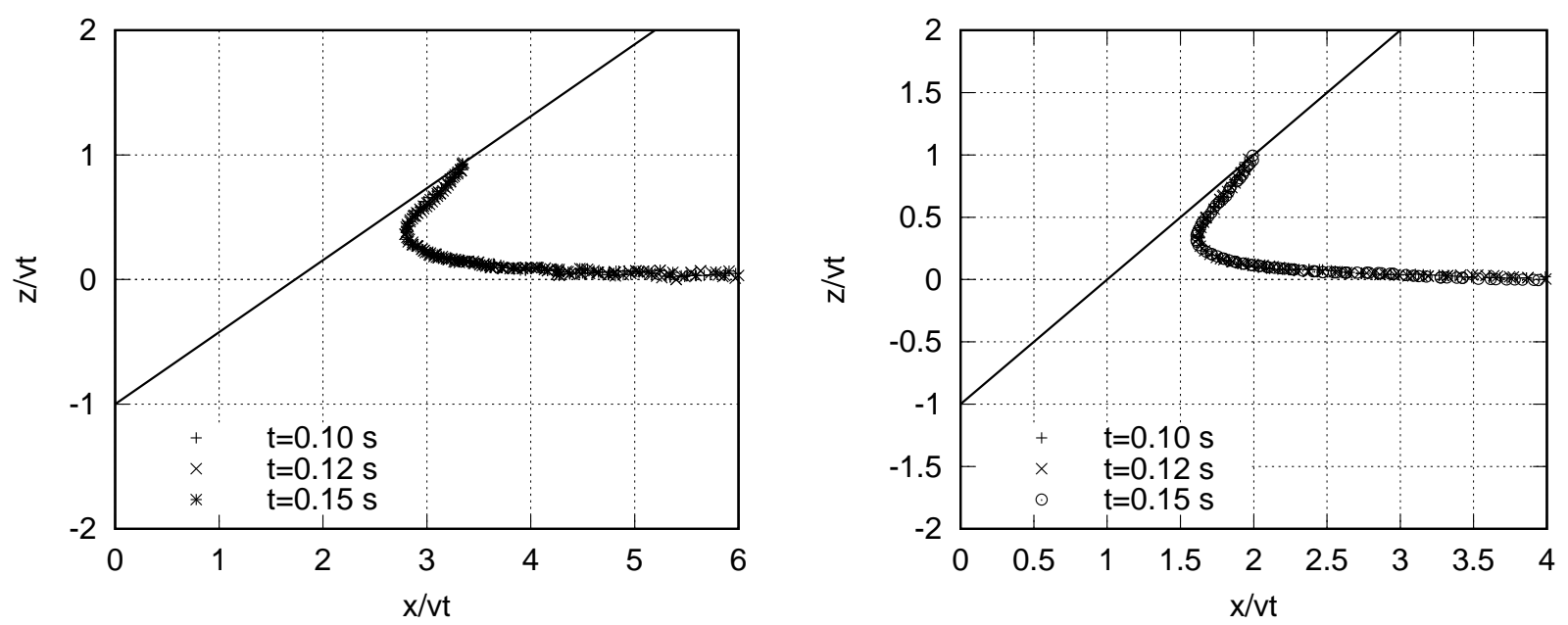

Figure 17: Sketch of the free surface profiles from the present model at different time instants. Left: $\phi=30^{\circ}$. Right: $\phi=45^{\circ}$.

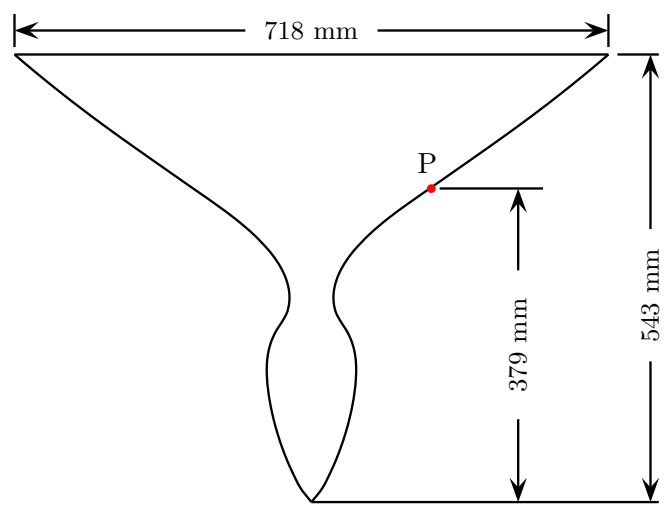

Figure 18: The geometry of the ship-like cross section and the position of the pressure sensor.

\subsubsection{Heave decay}

The heave motion during the free decay process is given in Fig. 24, where the results from the present model, a linear frequency domain BEM code and the experiment are included. It should be mentioned that the BEM result is for a full-scale FPA. In order to do a proper comparison, it is down-scaled by 1:10 in the time domain based on the following relation for the natural frequency $\omega_{r}$ :

$$
\omega_{r}=\sqrt{\frac{c}{m_{f}+A\left(\omega_{r}\right)}}
$$

where $c$ is the restoring coefficient and $A\left(\omega_{r}\right)$ is the added mass of the FPA at its natural frequency. From the experimental data in Fig. 24 the natural period of the FPA is $0.9 \mathrm{~s}$ in model scale, corresponding to $9 \mathrm{~s}$ in full scale. This is predicted well by the BEM model, while the present model slightly underestimates the natural period. This is probably due to underestimation of the added mass, since only a basic structure is considered in our model. However, the same geometry was also applied in the BEM model, but apparently it has less influence.

On the other hand, the decay speed predicted by the present model agrees well with the experimental data, while the BEM model produced a slower decay speed, i.e. the damping is underestimated. The reason is that the heave decay is related to both added mass and damping of the FPA as:

$$
\eta_{33}(t)=\hat{\eta}_{33} e^{-\delta t} \cos \left(\omega_{r} t\right)
$$




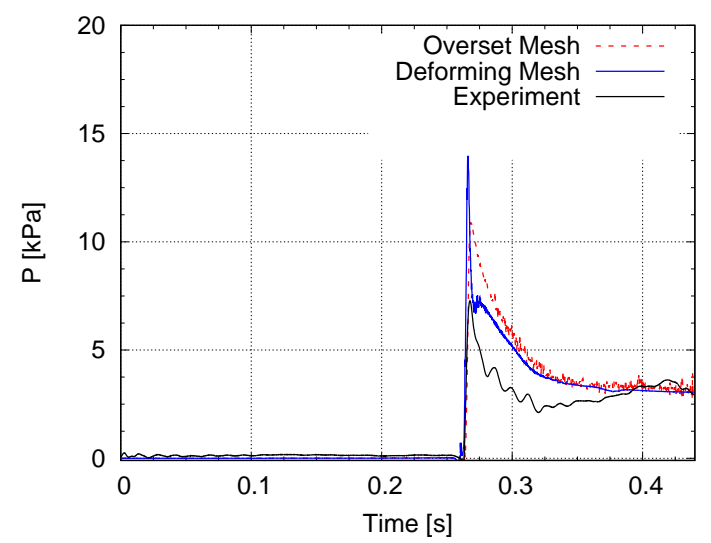

(a) The local pressure at $\mathrm{P}$.

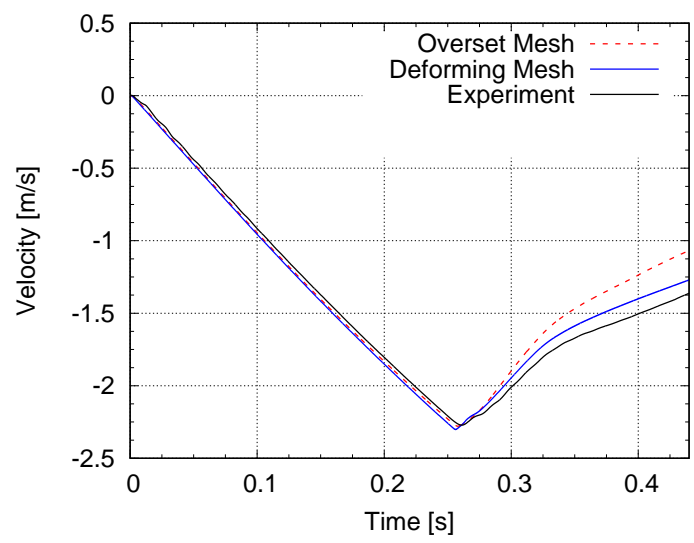

(c) The velcoty of the section.

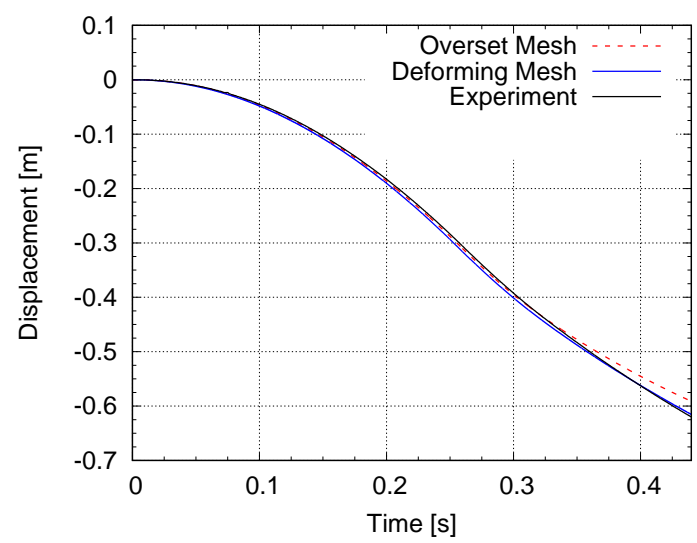

(b) The displacement of the section.

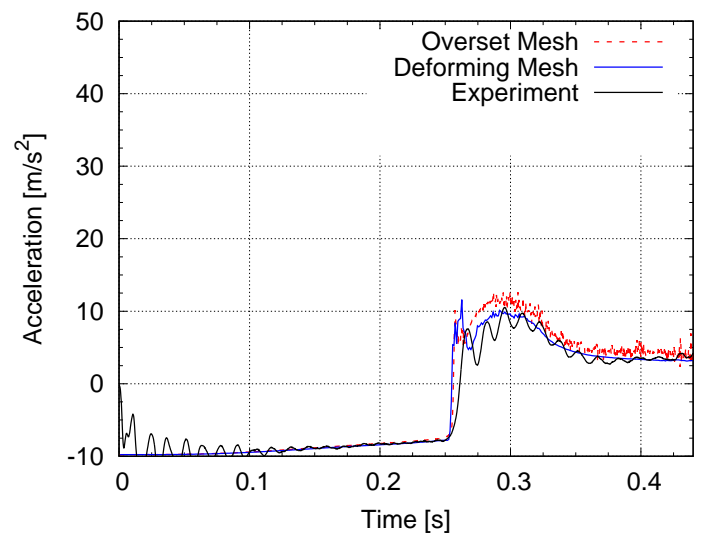

(d) The acceleration of the section.

Figure 19: Comparison of the numerical results using overset mesh and deforming mesh, and experimental results for the free-fall of a ship-like section.

where $\eta_{33}(t)$ is the heave motion, and $\hat{\eta}_{33}$ is the initial heave. $\delta$ controls the decay speed:

$$
\delta=-\frac{B\left(\omega_{r}\right)}{2\left(m_{f}+A\left(\omega_{r}\right)\right)}
$$

where $B\left(\omega_{r}\right)$ is the damping of the FPA at natural frequency. The damping force actually contains two parts, namely the potential damping as mentioned in Section 3.4 .3 and viscous damping. The potential damping can be correctly predicted by the BEM model since it is inertia dominated and related to the free surface motion. However, viscous damping is completely neglected in the BEM model, while it is accounted for in the present model.

\subsection{The hydrodynamic forces and motion of a model-scale lifeboat in regular waves}

The lifeboat has been an important component in large commercial ships and offshore oil and gas platforms. It needs to be designed in such a way that people on board can be evacuated efficiently in case of emergency. Recently a set of experiments were carried out in COAST laboratory, Plymouth University, UK on the response of a lifeboat in regular waves. In this section, this set of data will be used to examine our numerical model for prediction of wave interaction with floating bodies with complex 3D geometries. 


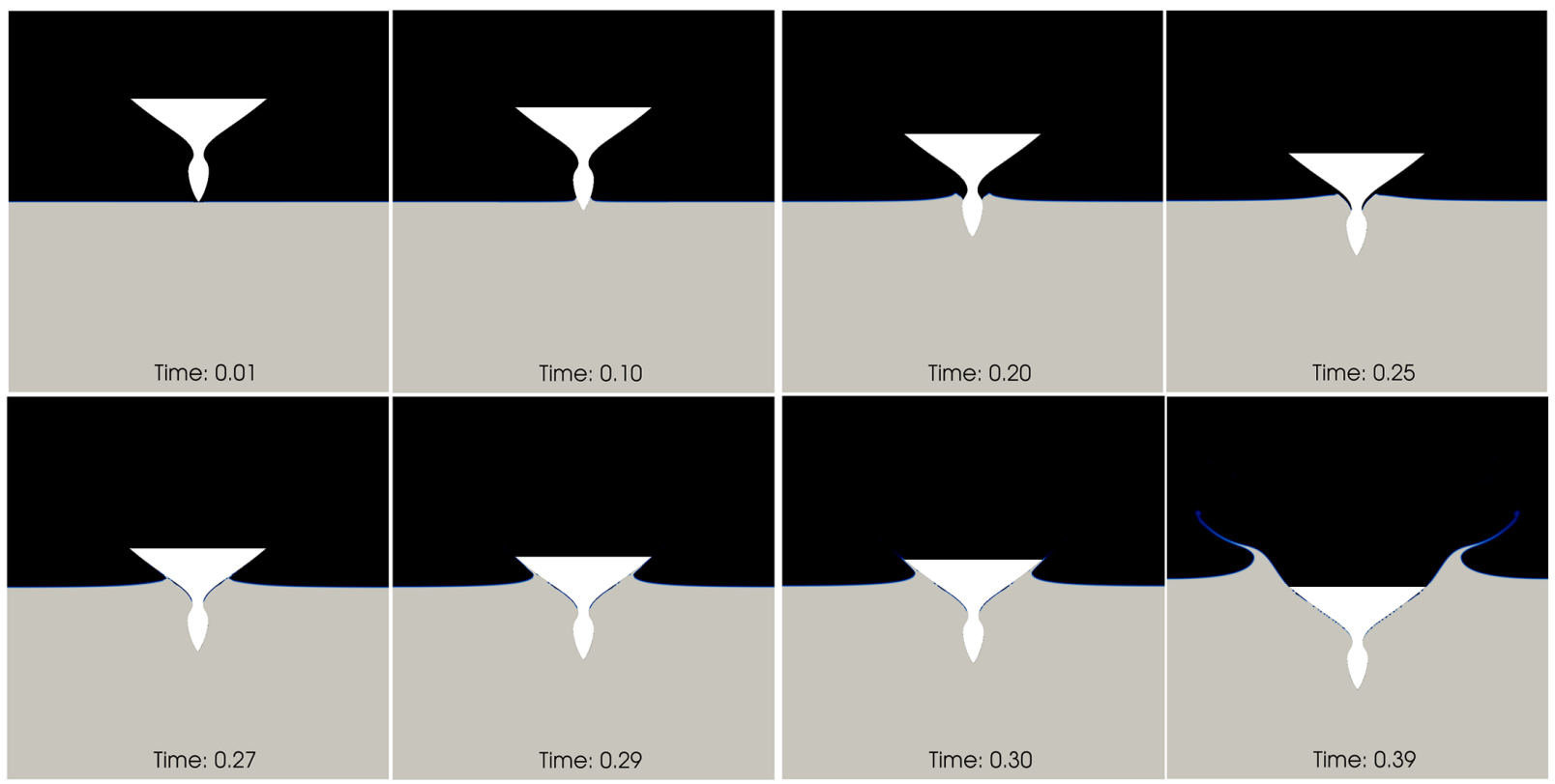

Figure 20: Free surface evolution during the water entry of a 2D ship-like section using overset mesh.

Table 3: The main particulars of the lifeboat model. $L_{p p}$ is the length between perpendicular, $B$ is the beam, $D$ is the draft, $\Delta$ is the displacement, LCG stands for longitudinal position of centre of gravity measured from the forward perpendicular, VCG stands for vertical position of centre of gravity measured from the baseline, $I$ is the moment of inertia of the lifeboat.

\begin{tabular}{cccccccccc}
\hline Item & $L_{p p}[\mathrm{~m}]$ & $B[\mathrm{~m}]$ & $D[\mathrm{~m}]$ & $\Delta[\mathrm{kg}]$ & LCG $[\mathrm{m}]$ & VCG $[\mathrm{m}]$ & $I_{x x}\left[\mathrm{kgm}^{2}\right]$ & $I_{y y}\left[\mathrm{kgm}^{2}\right]$ & $I_{z z}\left[\mathrm{kgm}^{2}\right]$ \\
\hline Value & 0.5 & 0.149 & 0.0535 & 1.36 & 0.2477 & 0.0535 & 0.002744 & 0.02176 & 0.02176 \\
\hline
\end{tabular}

\subsubsection{Experiments}

The experiments were performed in a narrow flume $30 \mathrm{~m}$ long and $0.6 \mathrm{~m}$ wide. The water depth was set to be $0.75 \mathrm{~m}$. The lifeboat model was downscaled from a full-scale lifeboat with a ratio of 1:20. The main particulars of the lifeboat model are given in Table 3. In the experiments it was positioned in the centre of the flume at a distance of $17 \mathrm{~m}$ from the wave-maker side. The selected wave condition is $H=0.016 \mathrm{~m}$ and $T=0.7 \mathrm{~s}$.

Two sets of experiments were conducted, one with a fixed lifeboat and one with a floating lifeboat. For the case with the fixed lifeboat, the hydrodynamic forces and moments were measured by a six-axis load cell, which was attached at the position of the centre of gravitation. Meanwhile 14 wave gauges were installed around the lifeboat to measure the wave elevation. For the case with the floating lifeboat, the lifeboat was moored by horizontal linear springs, whose stiffness was $6 \mathrm{~N} / \mathrm{m}$. The linear springs were actually connected to rigid vertical sticks, which were further fixed at the bow and stern of the lifeboat. No other constraints were added. Therefore, full six degree of freedom motion was allowed, and recorded by the Qualisys tracking system. Fig. 25 presents photographs of the experiments on both fixed and floating lifeboat. The positions of the wave gauges for the case with the fixed lifeboat are given in Fig. 26, where WG06 was deliberately neglected in the experiments.

\subsubsection{Setup of the numerical model}

In order to reproduce the experiments, a 3D numerical model is set up. The domain of the numerical wave tank is set to be $-10 L_{p p}<x<3 L_{p p}$, where the wave is propagating towards the bow of the lifeboat. The mooring lines are not directly resolved by the numerical model. Instead, the mooring forces are calculated and imposed in the rigid body motion equation (7)- 8), based on the stiffness of the spring line and the 
(a)

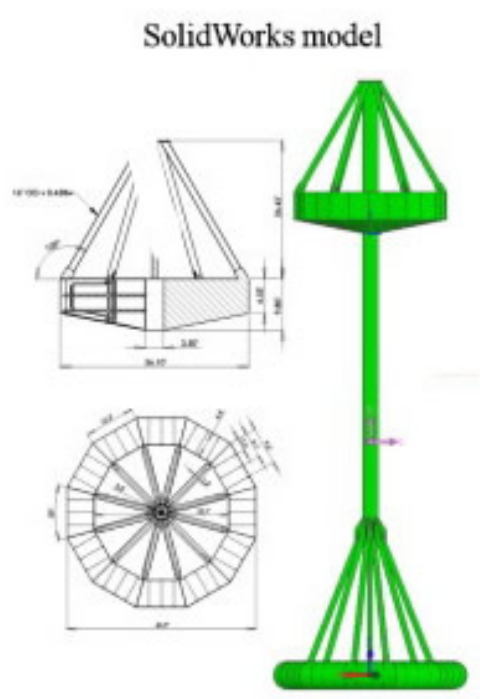

(b)

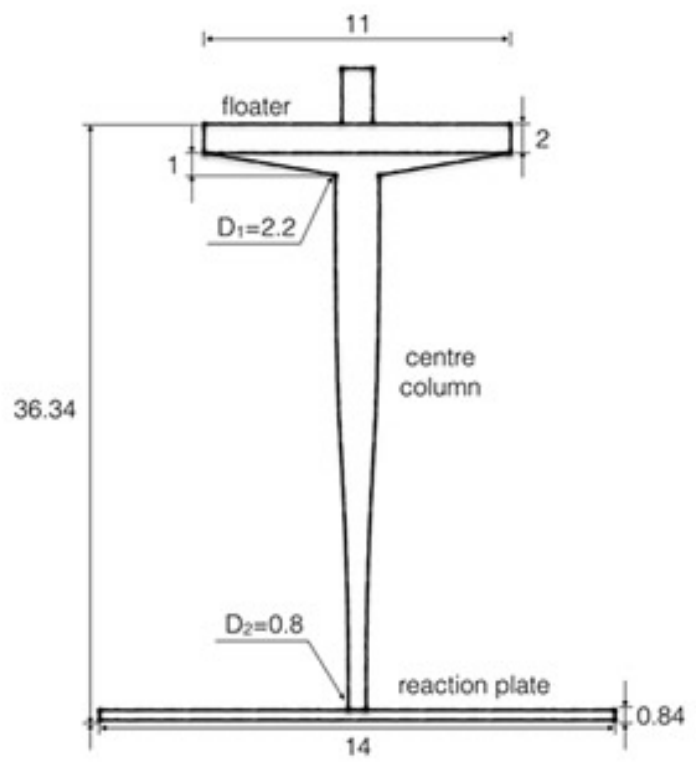

Figure 21: (a) Model-scale SRFPA in the experiment. (b) Dimension and geometry of the simplified model-scale SRFPA used in the numerical simulation (unit:cm).
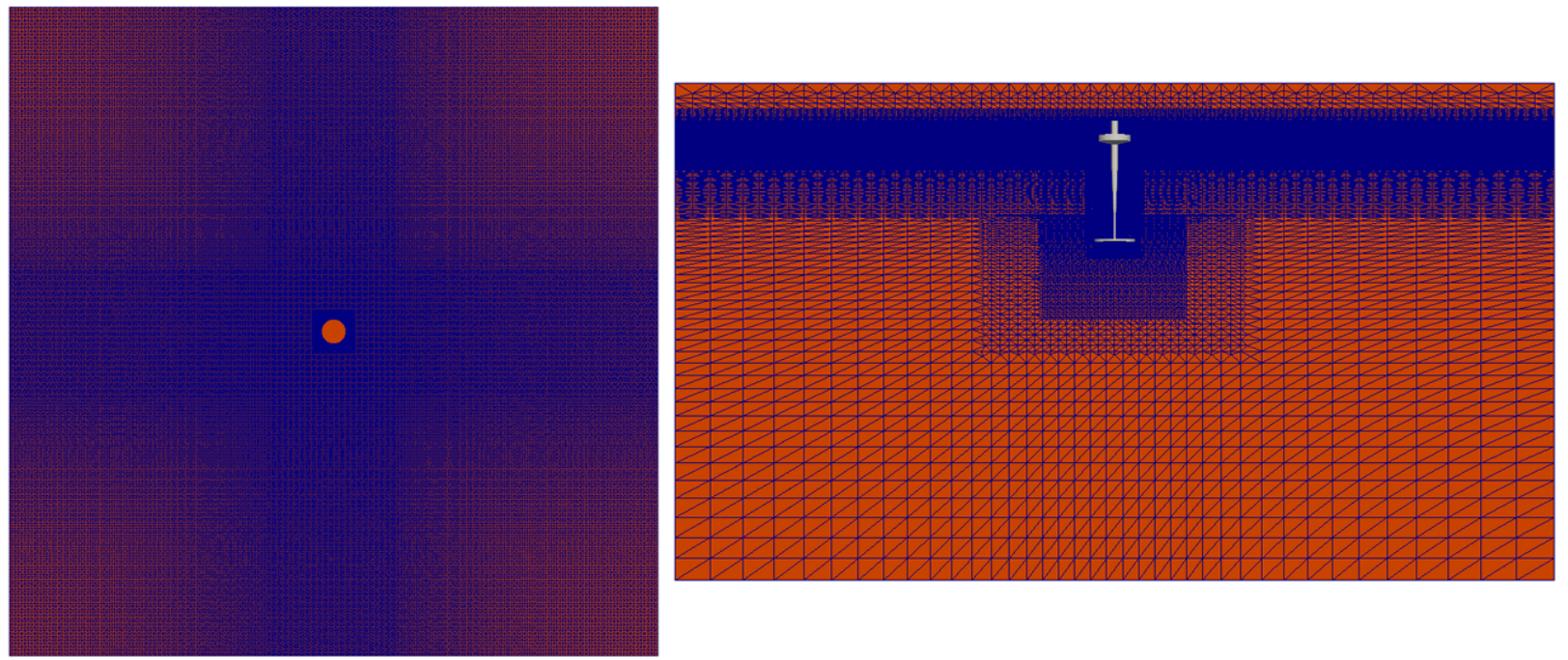

Figure 22: The computational mesh for the free decay of FPA. Left: top view on initial free surface plane. Right: side view on $y=0$ plane. 

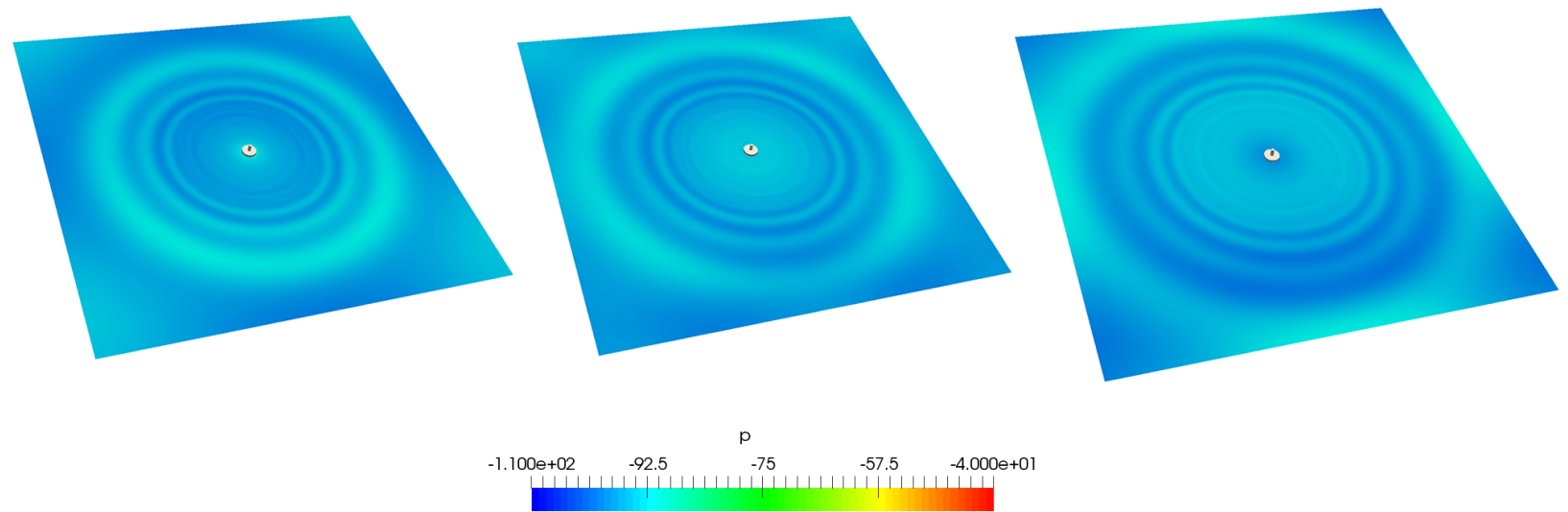

Figure 23: The hydrodynamic pressure contours on the free surface at $t=2.5, t=2.8$, and $t=3.0 \mathrm{~s}$.

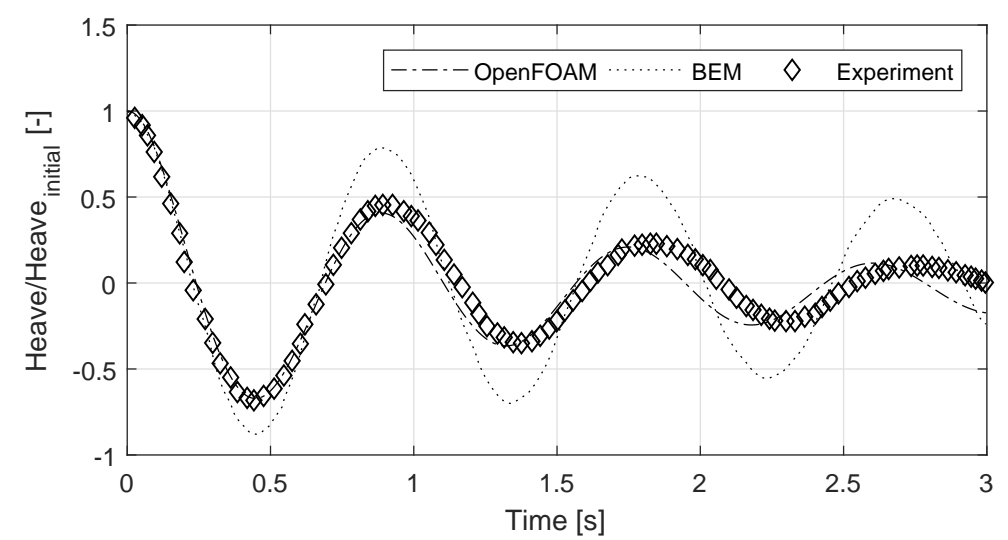

Figure 24: Heave decay test of the locked FPA from the present model (OpenFOAM), a frequency domain BEM code and the experimental data.

specified connecting points of the spring.

Fig. 27 presents the overset mesh used in the numerical model. A regular hexahedral mesh is generated in the domain as the background mesh, where the mesh near the lifeboat and in the vicinity of the free surface is refined significantly. This effectively ensures the mesh resolution for the incident and scattered free surface waves while ensuring that the computational cost remains at a reasonable level. The grid size in the body-fitted mesh is in general the same as in the refined area in the background mesh. In the region adjacent to the surface of the lifeboat, the mesh is further refined three times. The total number of grid cells including both the background layer and body fitted layer is approximately 1.4 million. In addition to the overset mesh, we also apply a dynamic mesh method for this case. The mesh resolution is quite similar since the background mesh used in snappyHexMesh is the same as the background mesh using the overset mesh method.

\subsubsection{Computational results}

Fig. 28 presents the hydrodynamic pressure field for the floating lifeboat case from $t=6.7 \mathrm{~s}$ to $t=7.2$ $\mathrm{s}$ using the overset mesh. The diffraction effect in this case is rather weak due to the relatively slender geometry of the lifeboat. Therefore, the incident waves are not significantly disturbed by the lifeboat and kept to the original wave shape. Fig. 29 further confirms the conclusion, where the surface elevation is given at various wave gauges for the case with the fixed lifeboat. It is observed that the surface elevation around the lifeboat is quite regular and similar to the incident waves. Regarding the performance of the numerical model, we conclude that quite good agreement is achieved between the numerical results using both mesh 

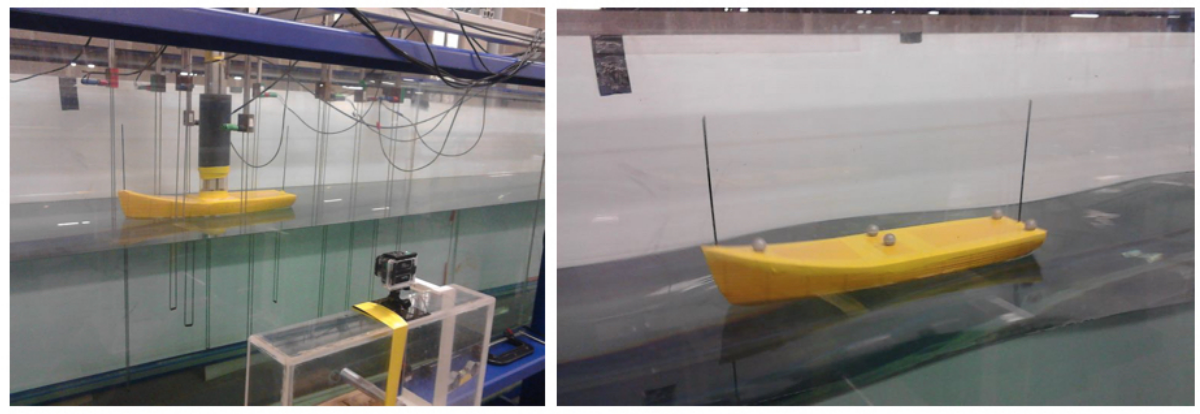

Figure 25: Photographs of the lifeboat model in the experiments. Left: fixed lifeboat. Right: floating lifeboat.

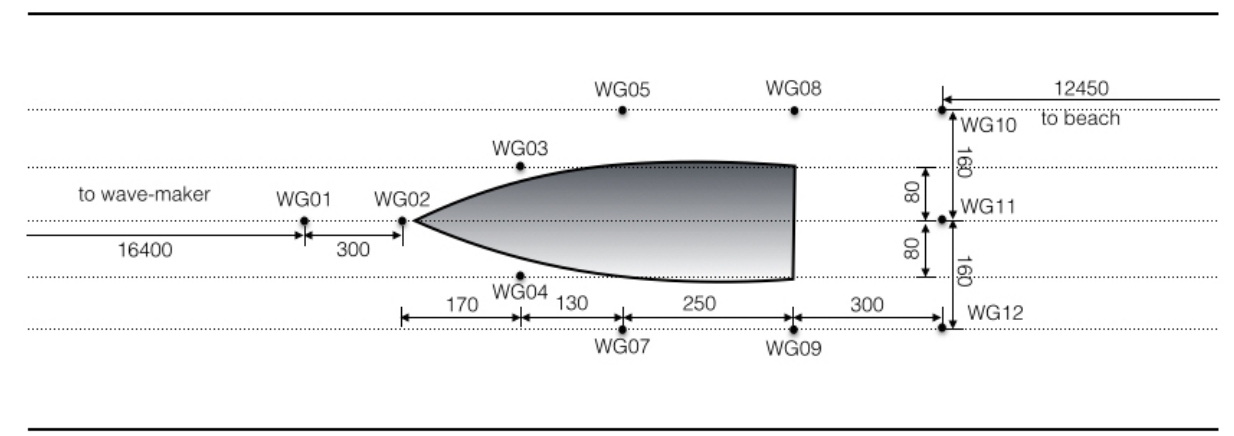

Figure 26: Position of the wave gauges and the lifeboat in the experiments for the case with fixed lifeboat (unit: $\mathrm{mm}$ ).

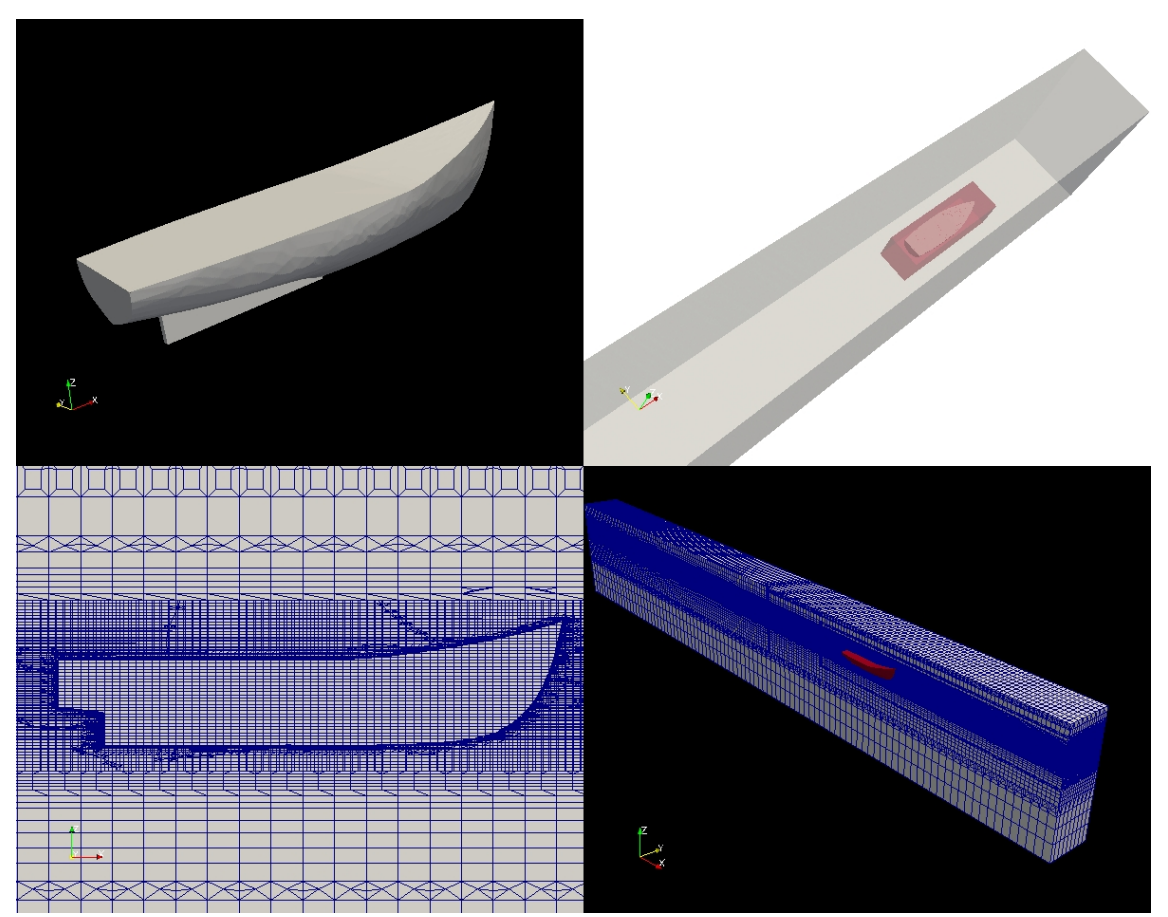

Figure 27: The computational mesh for the case with floating lifeboat. Upper left: the geometry of the lifeboat in the numerical model. Upper right: the overall domain size and the overlapping area around the lifeboat. Lower left: the mesh at the overlapping area where two layers of mesh are merged together. Lower right: an overview the mesh for the whole model. 


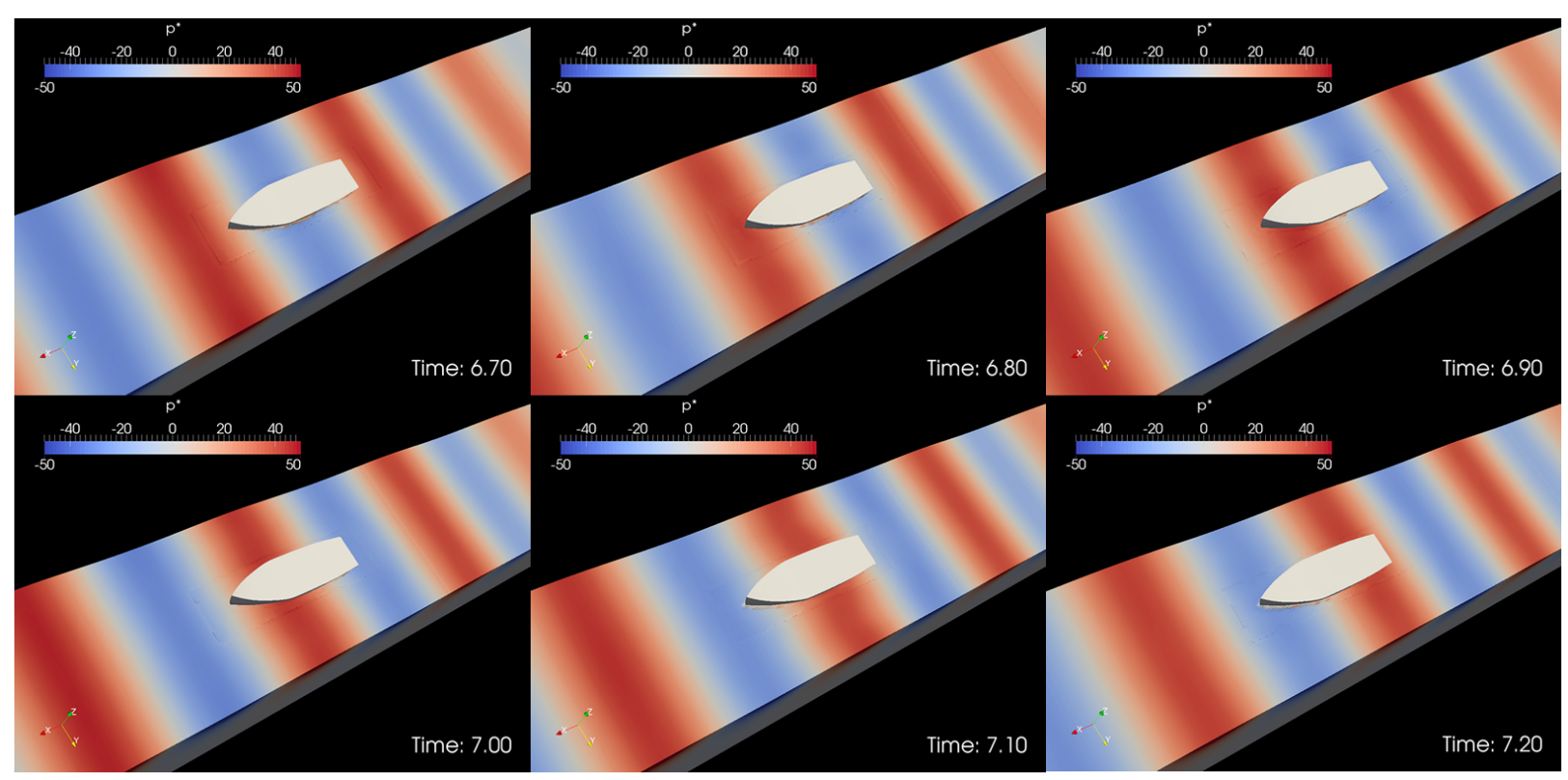

Figure 28: The contour for the excess pressure field near the floating lifeboat at different time instants.

methods and the experimental data. However, it is noticed that at the wave gauges close to the end of the numerical wave tank (e.g. wave gauge 11), the surface elevation is slightly underestimated, especially for the case with overset mesh method. This may be due to the numerical diffusion. With the propagation of waves, the wave energy is dissipated, which reduces the amplitude of waves. The diffusion appears in both time and spatial domain. For overset mesh, the interpolation process serves as another important source of the dissipation, as seen that it is more evident than the case with dynamic mesh.

The hydrodynamic forces on the fixed lifeboat are given in Fig. 30. Due to the symmetrical geometry and flow conditions, the force/moment for sway, roll and yaw should be zero. Therefore, we focus on the surge, heave forces and pitch moment. We notice that they are slightly but consistently overpredicted by both numerical models. The numerical model with dynamic mesh produces a generally more significant overestimation. Moreover, Fig. 31 presents the motion analysis of the floating lifeboat in surge, heave and pitch directions. Opposite to the hydrodyhamic forces, the motion is predicted well by the dynamic mesh method, while underestimated by the overset mesh method. For heave motion, the relative difference between the numerical model using overset mesh and the experimental data reaches $20 \%$, taking the experimental data as the reference solution. However, for surge and pitch motion, it is controlled to be within $15 \%$. In addition, when using the overset mesh method, we particularly observe a relatively pronounced initial error before the wave reaches the lifeboat, e.g. $0 \mathrm{~s}-2 \mathrm{~s}$ for the surge motion. This is due to non-still free surface in the initial simulation, resulted from the interpolation between the structured background mesh and the unstructured body-fitted mesh. This is likely to be eliminated when the incident wave arrives, since the non-zero value is relatively small comparing with the motion amplitudes in the quasi-steady period.

Furthermore, the experimental error due to blockage effect of the wave flume should also be considered. Actually the width of the flume is only four times of the lifeboat beam. The side wall effect might be amplified due to the relatively narrow width of the flume. Indeed, we find that the magnitude of the roll moment and motion are not negligible in experiments, while it is almost zero from the numerical model. This indicates that cross waves were generated in the laboratory tests, leading to the experimental error. However, the detailed uncertainty and error from the experiments are not known to the authors. 

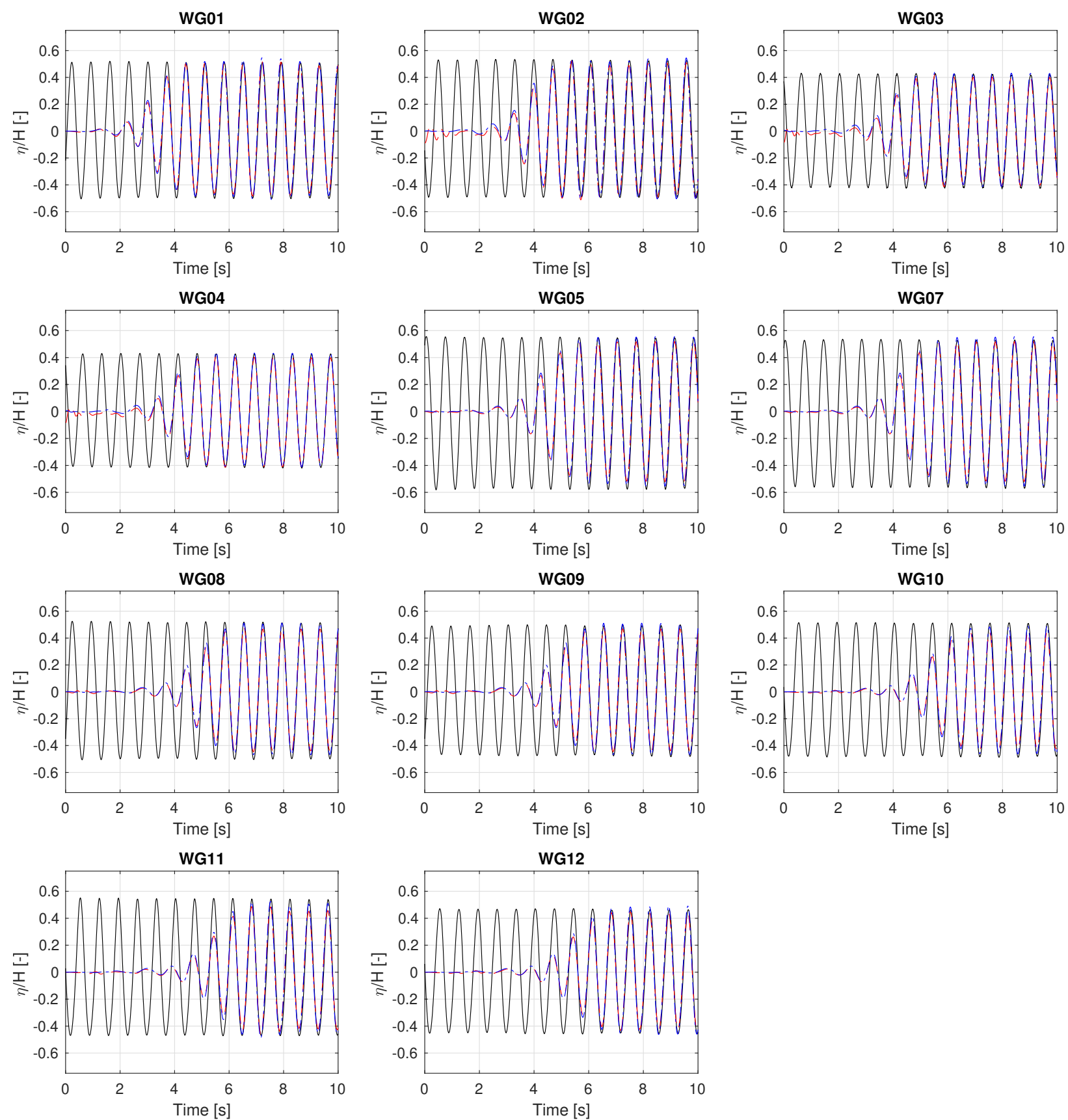

Figure 29: Comparison of the time series of the surface elevation between the numerical results and the experimental data from different wave gauges. The dashed red line represents the numerical results using overset mesh, the dashed blue line represents the numerical results using moving mesh and the black solid line is the experimental data. The incident wave condition is $H=0.016 \mathrm{~m}$ and $T=0.7 \mathrm{~s}$ and the positions of the wave gauges are given in Fig. 26. 

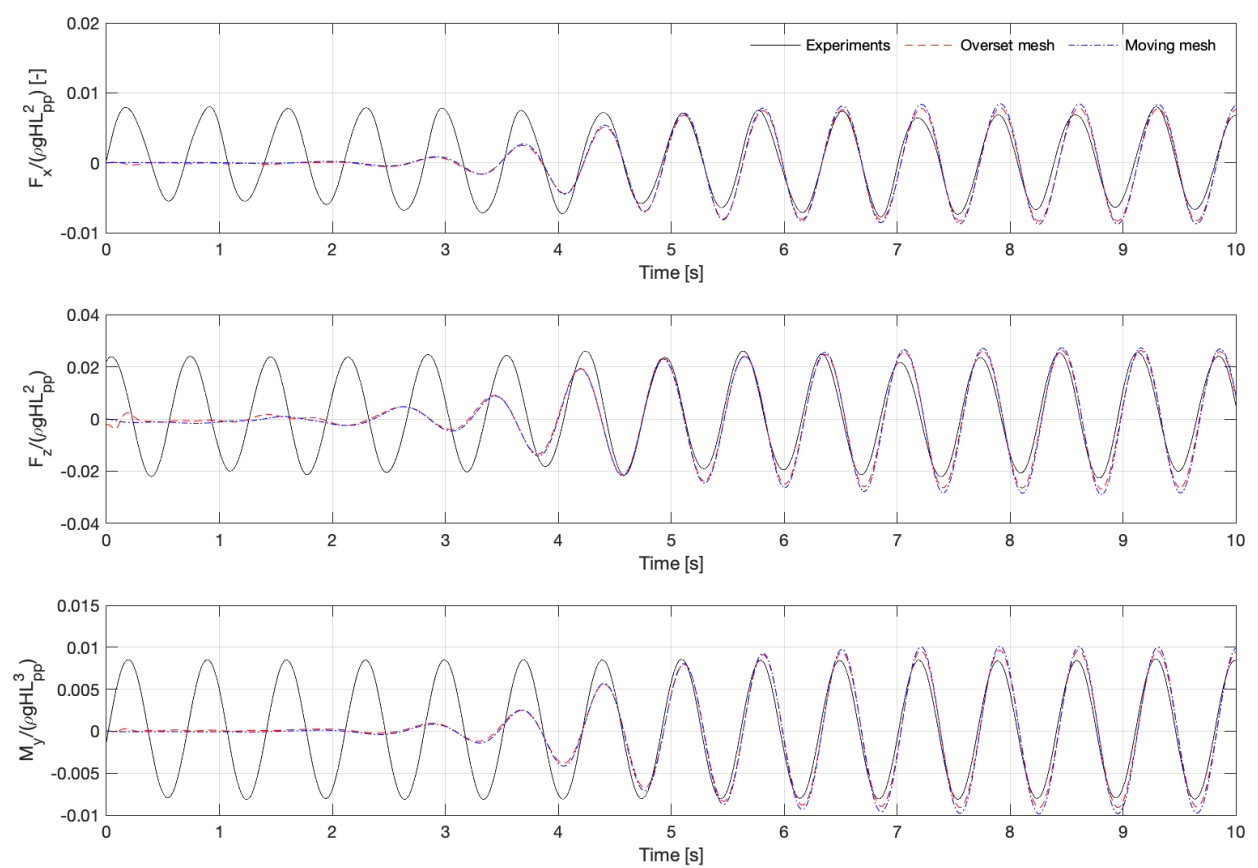

Figure 30: Comparison of the time series of hydrodynamic forces and moments between the numerical results and the experimental data for the fixed lifeboat in regular waves. The incident wave condition is $H=0.016 \mathrm{~m}$ and $T=0.7 \mathrm{~s}$.

\subsubsection{Comment on the computational cost and parallel efficiency}

3D seakeeping analysis is prohibitively expensive compared with frequency/time domain potential codes. Therefore, it is extremely important that a domain decomposition approach should work well with an overset mesh, so the computation can be carried out efficiently in parallel. Fig. 32 shows a numerical experiment on the efficiency of the parallel computation, where the computations are carried out for the lifeboat case. The test starts from $t=5 \mathrm{~s}$ and uses a fixed time step of $0.0001 \mathrm{~s}$. It is found that the speed-up is in general satisfactory, although with the increase of the CPU cores, the speed-up is likely to deviate from the ideal linear scaling. Actually, it takes about 48 hours for a $10 \mathrm{~s}$ simulation for this lifeboat case with $32 \mathrm{CPU}$ cores.

\section{Conclusions}

A numerical wave tank incorporating the new overset mesh functionality within OpenFOAM ${ }^{\circledR}$ has been presented. A number of benchmark test cases have been used to evaluate the effectiveness and accuracy of the overset mesh functionality for flow problems involving waves interacting with moving/floating structures, which include a 2D horizontal floating cylinder and a 2D box-shaped body with superstructure in regular waves, water entry of a $2 \mathrm{D}$ wedge and a ship-like section, the heave decay of a self-reacting floating point absorber and a model-scale lifeboat in regular waves. From these simulations, the following conclusions can be drawn:

1. The NWT based on the new overset approach can produce reasonably good results for wave interaction with floating structures with complex geometries or undergoing large amplitude of motions, as the numerical results in general agree well with the available experimental data. 

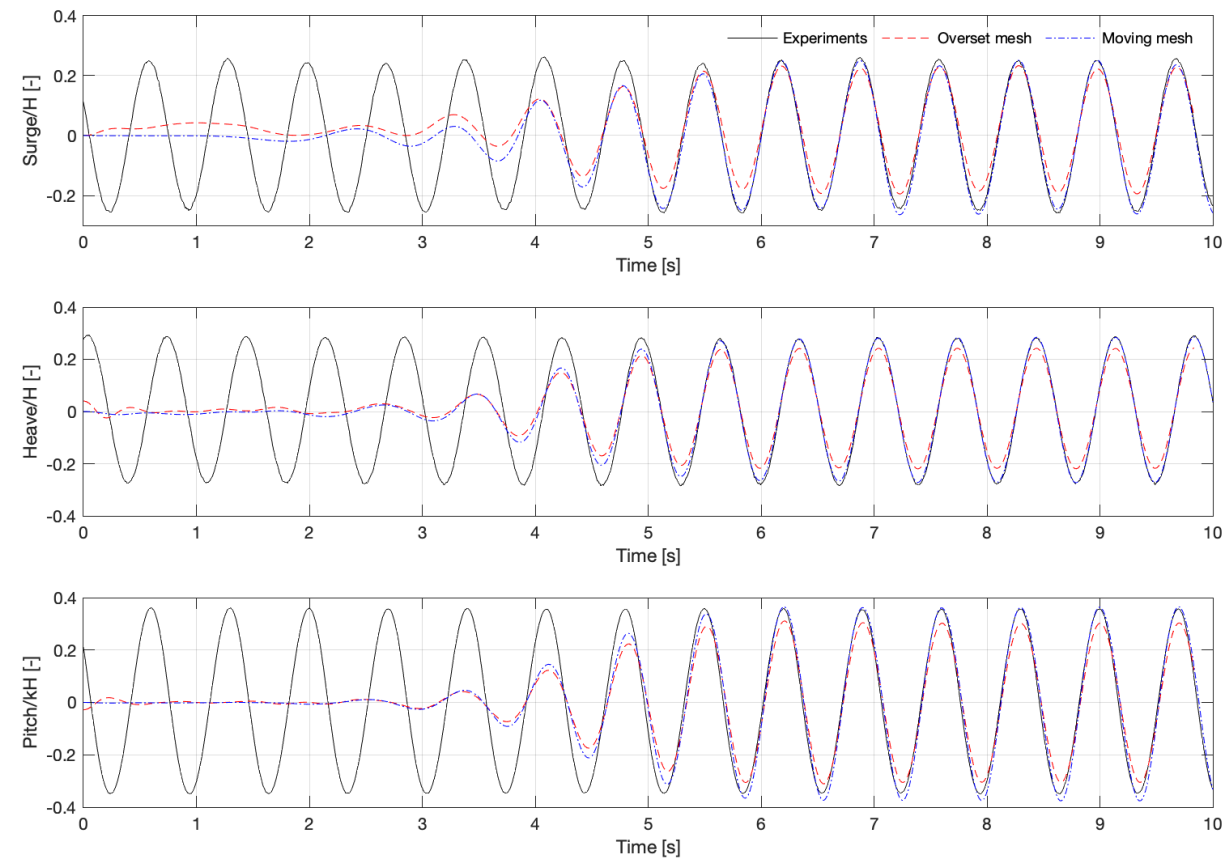

Figure 31: Comparison of the time series of the surge, heave and pitch motions between the numerical results and the experimental data for the floating lifeboat in regular waves. The incident wave condition is $H=0.016 \mathrm{~m}$ and $T=0.7 \mathrm{~s}$.

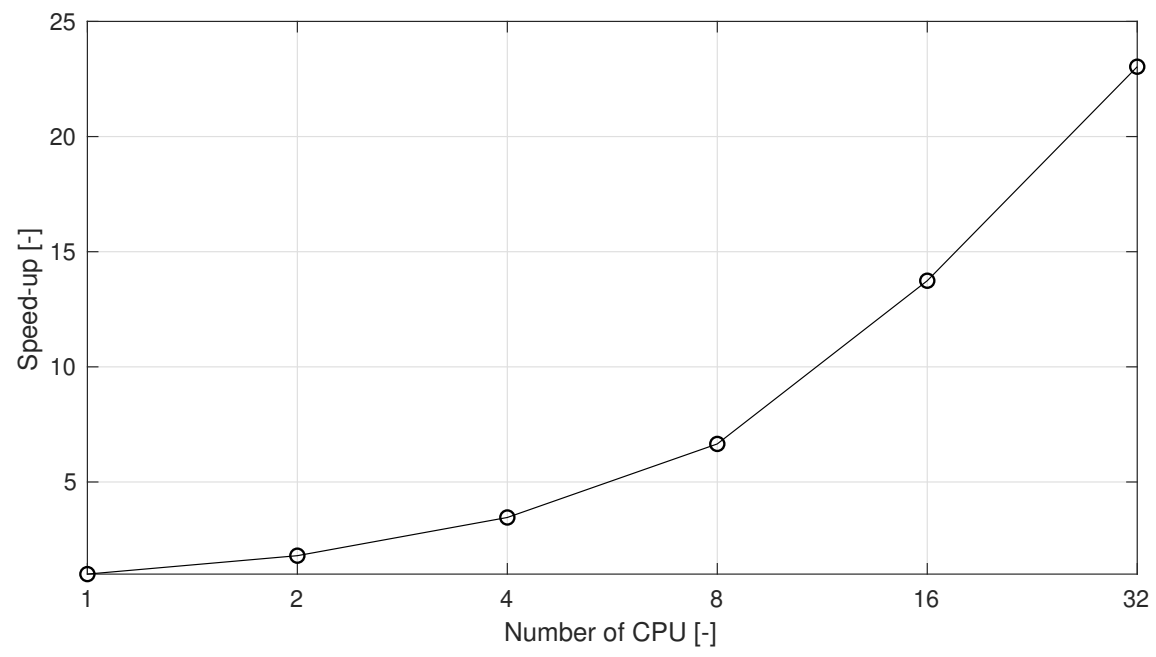

Figure 32: Test results for parallel computation efficiency. The time step is fixed of $0.0001 \mathrm{~s}$, and the computation starts from $t=5 \mathrm{~s}$ when the incident wave has reached the location of lifeboat. 
2. The overset mesh is a viable and favourable alternative for modelling moving bodies with large amplitude motions as demonstrated by the wedge slamming test case, which is difficult for the dynamic mesh approach.

3. For the cases which can be modelled by both overset mesh and dynamic mesh methods, the results are comparable with each other when a similar mesh resolution has been applied. However, with the current implementation, the overset method is in general less efficient than the dynamic mesh method.

4. The solution is shown to be not sensitive to the selection of the overlapping area, as long as the boundary of the overlapping area is sufficiently far away from the places where sharp gradients occur.

\section{Acknowledgements}

Dr. Nan Xie from Plymouth University is acknowledged for kindly providing the experimental data on the lifeboat tests. This research was supported by the Engineering and Physical Sciences Research Council (EPSRC), U.K. Project: A Zonal CFD Approach for Fully Nonlinear Simulations of Two Vessels in Launch and Recovery Operations, under Grant No. EP/N008839/1.

\section{References}

Bihs H, Kamath A. A combined level set/ghost cell immersed boundary representation for floating body simulations. International Journal for Numerical Methods in Fluids 2017;83(12):905-16. doi 10.1002/fld.4333

Brunswig J, Manzke M, Rung T. Explicit and implicit coupling strategies for overset grids. In: Proceedings of the 10th Symposium on Overset Composite Grids and Solution Strategies. Moffet Field, California: NASA AMES Research Center; 2010. .

Chan WM. Overset grid technology development at NASA Ames Research Center. Computers and Fluids 2009;38(3):496-503. doi $10.1016 / j$.compfluid.2008.06.009

Chan WM, Pandya SA. Advances in distance-based hole cuts on overset grids. In: 22nd AIAA Computational Fluid Dynamics Conference. 2015. p. 3425.

Chella MA, Bihs H, Myrhaug D. Characteristics and profile asymmetry properties of waves breaking over an impermeable submerged reef. Coastal Engineering 2015;100:26-36.

Dobrovol'Skaya Z. On some problems of similarity flow of fluid with a free surface. Journal of Fluid Mechanics 1969;36(4):80529.

Druyor CT. Advances in parallel overset domain assembly. Ph.D. thesis; The University of Tennessee at Chattanooga; 2016

Gatin I, Vukčević V, Jasak H, Seo J, Rhee SH. CFD verification and validation of green sea loads. Ocean Engineering 2018;148(August 2017):500-15. doi 10.1016/j.oceaneng.2017.10.026

Higuera P, Lara JL, Losada IJ. Realistic wave generation and active wave absorption for Navier-Stokes models. Application to OpenFOAM®. Coastal Engineering 2013;71:102-18. doi $10.1016 / \mathrm{j}$. coastaleng.2012.07.002.

Issa RI. Solution of the implicitly discretised fluid flow equations by operator-splitting. Journal of Computational Physics 1986;62(1):40-65. URL: http://www.sciencedirect.com/science/article/pii/0021999186900999 doi 10.1016/ 0021-9991(86) 90099-9.

Iturrioz A, Guanche R, Lara J, Vidal C, Losada I. Validation of openfoam $\AA$ for oscillating water column three-dimensional modeling. Ocean Engineering 2015;107:222-36.

Jacobsen NG, Fuhrman DR, Fredsoe J. A wave generation toolbox for the open-source CFD library: OpenFoam (R). International Journal for Numerical Methods in Fluids 2012;70(9):1073-88. doi 10.1002/fld.2726

Kamath A, Chella MA, Bihs H, Arntsen $\varnothing$ A. Cfd investigations of wave interaction with a pair of large tandem cylinders. Ocean Engineering 2015;108:738-48.

Kristiansen D. Wave induced effects on floaters of aquaculture plants. Ph.d. thesis; Norwegian University of Science and Technology; 2010.

Ma ZH, Qian L, Martínez-Ferrer PJ, Causon DM, Mingham CG, Bai W. An overset mesh based multiphase flow solver for water entry problems. Computers and Fluids 2018;172(0):689-705.

Márquez Damián S. An extended mixture model for the simultaneous treatment of short and long scale interfaces. Ph.d. thesis; UNIVERSIDAD NACIONAL DEL LITORAL; 2013.

Martínez Ferrer PJ, Causon DM, Qian L, Mingham CG, Ma ZH. A multi-region coupling scheme for compressible and incompressible flow solvers for two-phase flow in a numerical wave tank. Computers and Fluids 2016;125:116-29. doi 10 . 1016/j.compfluid.2015.11.005.

Martínez-Ferrer PJ, Qian L, Ma Z, Causon DM, Mingham CG. Improved numerical wave generation for modelling ocean and coastal engineering problems. Ocean Engineering 2018;152:257-72. doi $10.1016 / \mathrm{j}$.oceaneng. 2018.01.052

Mei X, Liu Y, Yue DK. On the water impact of general two-dimensional sections. Applied Ocean Research 1999:21(1):1-15.

Ong MC, Kamath A, Bihs H, Afzal MS. Numerical simulation of free-surface waves past two semi-submerged horizontal circular cylinders in tandem. Marine Structures 2017;52:1-14. doi $10.1016 / j . m a r s t r u c .2016 .11 .002$ 
Palm J, Eskilsson C, Moura Paredes G, Bergdahl L. Coupled mooring analysis of floating wave energy converters using CFD. International Journal of Marine Energy 2016;16:83-99. doi 10.1016/j.ijome.2016.05.003.

Paolo BD, Lara JL, Barajas G, Paci A, Losada IJ. Numerical analysis of wave and current interaction with moored floating bodies using overset method. In: Proceedings of the ASME 2018 37th International Conference on Ocean, Offshore and Arctic Engineering. 2018.

Park JC, Kim MH, Miyata H, Chun HH. Fully nonlinear numerical wave tank (NWT) simulations and wave run-up prediction around 3-D structures. Ocean Engineering 2003;30(15):1969-96. doi 10.1016/S0029-8018(03) 00041-6

Patankar SV, Spalding DB. A calculation procedure for heat, mass and momentum transfer in three-dimensional parabolic flows. In: Numerical Prediction of Flow, Heat Transfer, Turbulence and Combustion. Elsevier; 1983. p. 54-73.

Paulsen BT, Bredmose H, Bingham H, Jacobsen NG. Forcing of a bottom-mounted circular cylinder by steep regular water waves at finite depth. Journal of Fluid Mechanics 2014;755:1-34. doi 10.1017/jfm.2014.386

Vukčević V, Jasak H, Malenica Š. Decomposition model for naval hydrodynamic applications, Part I: Computational method. Ocean Engineering 2016;121:76-88. doi http://dx.doi.org/10.1016/j.oceaneng.2016.05.021

Windt C, Davidson J, Akram B, Ringwood JV. Performance assessment of the overset grid method for numerical wave tank experiments in the openfoam environment. In: Proceedings of the ASME 2018 37th International Conference on Ocean, Offshore and Arctic Engineering. 2018. .

Yang F, Shi W, Zhou X, Guo B, Wang D. Numerical investigation of a wave glider in head seas. Ocean Engineering 2018;164:127 -38. doi https://doi.org/10.1016/j.oceaneng.2018.06.017

Yu YH, Li Y. Reynolds-Averaged Navier-Stokes simulation of the heave performance of a two-body floating-point absorber wave energy system. Computers and Fluids 2013;73:104-14. doi 10.1016/j.compfluid.2012.10.007

Zhao X, Hu C. Numerical and experimental study on a 2-D floating body under extreme wave conditions. Applied Ocean Research 2012;35:1-13. doi 10.1016/j.apor.2012.01.001 